\title{
Method of Moment Analysis of Carbon Nanotubes Embedded in A Lossy Dielectric Slab Using A Multilayer Dyadic Green's Function
}

\author{
Sumitra Dey, Student Member, IEEE, Deb Chatterjee Sr., Member, IEEE, Edward J. Garboczi, and Ahmed M. \\ Hassan Sr., Member, IEEE
}

\begin{abstract}
Modeling the electromagnetic response of carbon nanotube (CNT) reinforced composites is inherently a threedimensional (3D) multi-scale problem that is challenging to solve in real-time for nondestructive evaluation applications. This article presents a fast and accurate full-wave electromagnetic solver based on a multi-layer dyadic Green's function approach. In this approach, we account for the effects of the dielectric slab, where the CNTs are embedded, without explicitly discretizing its interfaces. Due to their large aspect ratios, the CNTs are modeled as arbitrary thin wires (ATWs), and the method of moment (MoM) formulation with distributed line impedance is used to solve for their coupled currents. The accuracy of the inhouse solver is validated against commercial method of moment (MoM) and finite element method (FEM) solvers over a broad range of frequencies (from $1 \mathrm{GHz}$ to $10 \mathrm{THz}$ ) and for a wide range of dielectric slab properties. Examples of $100 \mathrm{~nm}$ long vertical and horizontal CNTs embedded in a $1 \mu \mathrm{m}$ thick lossy dielectric substrate are presented. The in-house solver provides more than $50 \times$ speed up while solving the vertical CNT, and more than $570 \times$ speed up while solving the horizontal CNT than a commercial MoM solver over the GHz to THz frequency range.
\end{abstract}

Index Terms-Arbitrary thin wire (ATW), carbon nanotubes (CNTs), electric field integral equation (EFIE), method of moment (MoM), multilayer dyadic Green's function, Sommerfeld integrals (SI).

\section{INTRODUCTION}

$\mathbf{O}$ VER the past decade, carbon nanotube (CNT) reinforced composites have been used in a wide range of applications including automotive and aerospace materials, electro-mechanical actuation and sensing, packaging, adhesives, conductive ink, coatings, electromagnetic interference (EMI) shielding and many more [1]-[5]. The unique carbon atom arrangements of metallic CNTs provides them with a unique high electrical conductivity [1], [2]. The inclusion of a small volume fraction of CNT fillers

This paragraph of the first footnote will contain the date on which you submitted your paper for review.

This work was supported in part by the NIST "Multi-Scale Computational Modeling of Carbon Nanostructure Composites" under Grant 70NANB15H285 and in part by NSF CRI Award 1629908 "II-NEW: Experimental Characterization and CAD Development Testbed for Nanoscale Integrated Circuits." (Corresponding author: Sumitra Dey.)

S. Dey, D. Chatterjee, and A. M. Hassan are with the Department of Computer Science and Electrical Engineering, University of Missouri-Kansas City, Kansas City, MO 64110 USA (e-mail: sumitra.dey@mail.umkc.edu; ChatD@umkc.edu; hassanam@umkc.edu).

E. J. Garboczi is with the Material Measurement Laboratory, Applied Chemicals and Materials Division, National Institute of Standards and Technology, Boulder, CO 80305 USA (e-mail: edward.garboczi@nist.gov). can produce light-weight, durable, ultra-thin and flexible composites with high dielectric constants [6], [7].

Several factors control the electromagnetic response of CNT composites, such as the spatial distribution of CNTs [8]-[10], CNT volume fraction [11], average length of embedded CNTs [12], [13], interaction among CNTs [13], [14], conductivity of CNTs (single wall/multi wall) [11], [15], waviness of CNTs (nearly straight to highly crumpled) [16], [17], CNT interaction with matrix, matrix properties, and dimensions [18], [19]. Thus, finding an efficient method capable of accurately quantifying the interactions among CNTs, interactions of CNTs with the embedding layers, and with the incident electromagnetic excitation is critical for understanding and optimizing the electromagnet response of CNT reinforced composites. This will help guiding the process of composite fabrication and monitoring composite health during their service life and check for any structural degradation [5], [20].

To predict the effective permittivity and permeability of composite materials, many theories have been proposed and extended such as the Waterman-Truell [21]-[24], and Maxwell-Garnett (MG) approximations [25], [26]. These theories are commonly known as the dilute limit effective medium approximation (EMA) theory which assume low filler density and operate at a low frequency range. EMA theories do not incorporate the effects of the finer distribution of the fillers, interaction among fillers when in close proximity, and filler interaction with the embedding matrix. For example, Hassan et al. recently used two independent commercial fullwave solvers, the method of moment (MoM) based FEKO ${ }^{1}$, and the finite element method (FEM) based CST Microwave Studio (MWS), to verify that even at small volume fractions, dilute limit EMA fails to account for the strong interactions between adjacent CNTs, and differs significantly from the fullwave simulations, especially at higher terahertz frequencies [27].

Full wave electromagnetic analysis ensures high accuracy of the solution provided the system under observation is discretized with an adequate size and number of mesh elements. The main bottleneck in discretizing CNT composites

\footnotetext{
${ }^{1}$ Certain commercial equipment, instruments, or materials are identified in this paper to foster understanding. Such identification does not imply recommendation or endorsement by the National Institute of Standards and Technology, nor does it imply that the materials or equipment identified are necessarily the best available for the purpose.
} 
arises due to their multiscale nature. The CNTs are high aspect ratio pipe-like structures whose diameter varies from a few $\mathrm{nm}$ to hundreds of $\mathrm{nm}$, and lengths varies from hundreds of $\mathrm{nm}$ to several $\mu \mathrm{m}$. Embedding matrix thicknesses vary from several $\mu \mathrm{m}$ to several $\mathrm{mm}$, and lateral extents may go beyond the $\mathrm{cm}$ range. In $1 \mathrm{~cm}^{3}$ composite volume, the number of CNTs may vary from several thousands to billions. Thus, volume/surface discretization of the entire composite structure tremendously increases the computational cost. For example, Wang et al. used finite element analysis (FEA) based COMSOL Multiphysics software to study the effects of $\mathrm{CNT}$ orientation on the resulting relative permittivity of CNT composites [8]. They modeled multiple $600 \mathrm{~nm}$ long CNTs inside a three dimensional (3D) unit cell $(1.5 \mu \mathrm{m} \times 1.5 \mu \mathrm{m} \times$ $0.6 \mu \mathrm{m})$ and applied periodic boundary condition to simulate the composite. According to that study, the tetrahedral mesh per unit cell increased from 413,702 to 1,903,693 elements, when the number of embedded CNTs were increased from 5 to 40 , with approximately a 134 -fold increase in computational time. Hassan et al. have also discussed the increase in computational time necessary to accurately simulate a realistic distribution of a large number of 3D CNTs [27] .

In this paper, we propose an alternate full wave approach for analyzing multiscale CNT composites that reduces the computation time by several orders of magnitude compared to a commercial full wave solver. The approach studied herein adopts the following strategies.

1) To eliminate the need of volume/surface discretization of the dielectric slab or substrate interfaces, we use a multilayer dyadic Green's function (DGF) formulation where the substrate is simulated as a finite thickness lossy dielectric slab with infinite lateral extent [28], [29].

2) The embedded high-aspect ratio CNTs are modeled as one dimensional (1D) arbitrary thin wires (ATW) and discretized into 1D segments [13], [16]. This eliminates a huge number of surface/volume mesh elements required for the explicit modeling of 3D cylindrical CNTs.

3) The ATW CNTs are assigned distributed frequency dependent complex impedances [30], and their corresponding induced currents are calculated using the method of moment (MoM) based on the electric field integral equation (EFIE) technique [31].

The DGFs are defined for lossy three layer stratified media, which include a special class of integrals known as the Sommerfeld integrals (SI) that have no direct analytical solutions [32], [33]. The accuracy and the computational speed of the solver depends directly on the efficiency and accuracy of the SI evaluation method. This work proposes a modified semianalytical approach to evaluate the SIs associated with the DGFs, which addresses the SI instability issues in multiscale layered media and employs an adaptive elliptic contour deformation technique to avoid integrand singularities [34][36]. We also propose an efficient way to evaluate the SI tail in appropriate closed forms that circumvents time consuming slow convergence issues [37]. The in-house full wave 1D MoM solver was developed on the MATLAB [38] platform and was

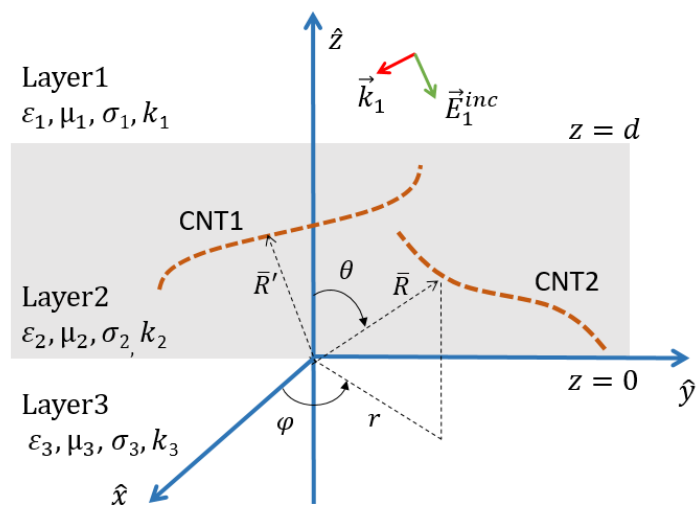

Fig. 1: General planar stratified three layer structure with embedded CNTs in layer 2.

validated in two phases. First, the DGFs are validated using an infinitesimal embedded dipole for a vast range of embedding media properties from $1 \mathrm{GHz}$ to $10 \mathrm{THz}$ against the planar substrate solver of FEKO [39]. Next, the absorption power spectrum of a horizontal and a vertical embedded CNT is computed using the in-house solver and is validated against the 3D MoM solver FEKO [39], and 3D FEM solver CST MWS [40]. The comparison shows that the in-house solver is more efficient and several orders of magnitude faster than the commercial solvers which requires explicit discretization of the 3D CNT surface and/or the interfaces of the dielectric slab. This demonstrates the potential of the in-house solver for realtime interpretation of the scattering measurements typically performed during non-destructive evaluation (NDE) of CNT reinforced composites, or any layered composites with wirelike fillers.

\section{Formulation}

The formulation of the proposed full wave MoM solver is outlined in this section. The problem is defined by electric field integral equations (EFIE) with proper boundary conditions pertaining to the embedded CNTs. Detailed expressions of the dyadic Green's functions (DGF) are provided and their solution with a semi-analytic approach is discussed. Different interpolation techniques and look-up tables are implemented to reduce the computation time for the MoM matrices.

\section{A. EFIE for Embedded Arbitrarily Thin CNTs}

A generic case of a lossy three-layer stratified planar structure is illustrated in Fig. 1, where the CNTs are embedded in layer 2, which has a finite thickness $(d)$ and is backed by two other lossy semi-infinite layers (layer 1 and layer 3 ). The layered structure is illuminated by a plane wave excitation $\left(\bar{E}_{1}^{i n c}\right)$ incident on the top of layer 1 . The interface between layer 1 and layer 2 is defined by the $z=d$ plane, and the interface between layer 2 and layer 3 is defined by the $z=0$ plane. The layers have real values of permittivity $\varepsilon_{i}=\varepsilon_{0} \varepsilon_{r i}$, permeability $\mu_{i}=\mu_{0} \mu_{r i}$, and conductivity $\sigma_{i}$, where the layer number is given by $i=1,2,3$, and $\varepsilon_{0}$ and $\mu_{0}$ are the vacuum permittivity and permeability, respectively. Thus the associated propagation constants in these three regions 
TABLE I: Parameter Range for the Multiscale CNT Composite Problem

\begin{tabular}{|l|l|l|}
\hline \multicolumn{3}{|c|}{ Electrical Properties of Embedding Media } \\
$\varepsilon_{r i}=1$ to $20, \mu_{r i}=1$ to $20, \sigma_{i}=1 \mathrm{~S} / \mathrm{m}$ to $20 \mathrm{~S} / \mathrm{m}$, for $\mathrm{i}=1,2,3$ \\
\hline \hline \multicolumn{3}{|c|}{ Operating frequency $(f)=1 \mathrm{GHz}-10 \mathrm{THz}$} \\
\hline Physical lengths & $f=1 \mathrm{GHz}$ & $f=10 \mathrm{THz}$ \\
\hline free-space wavelength $\left(\lambda_{0}\right)$ & $0.3 \mathrm{~m}$ & $3 \times 10^{-5} \mathrm{~m}$ \\
$\left|\bar{R}-\bar{R}^{\prime}\right|_{\min }=r_{c n t}=0.61 \mathrm{~nm}$ & $2.033 \times 10^{-9} \lambda_{0}$ & $2.033 \times 10^{-5} \lambda_{0}$ \\
$\left|\bar{R}-\bar{R}^{\prime}\right|_{\max }=1 \mathrm{~cm}$ & $0.033 \lambda_{0}$ & $333.33 \lambda_{0}$ \\
$d_{\text {min }}=10 \mathrm{~nm}$ & $3.33 \times 10^{-8} \lambda_{0}$ & $3.33 \times 10^{-4} \lambda_{0}$ \\
$d_{\max }=2 \mathrm{~mm}$ & $6.66 \times 10^{-3} \lambda_{0}$ & $66.66 \lambda_{0}$ \\
\hline
\end{tabular}

are complex and expressed as $k_{i}=\sqrt{\omega^{2} \mu_{i} \varepsilon_{i}-j \omega \mu_{i} \sigma_{i}}$, where $\omega$ is the angular operating frequency and $j=\sqrt{-1}$. The high aspect ratio CNTs are modeled as one-dimensional arbitrary thin wires (ATW) as validated previously in [13], [16]. Thus, the induced currents on the CNTs will have only an axial component and no circumferential variation. The CNTs are characterized by a Drude-like surface conductivity $\left(\sigma_{c n t}\right)$ as described in [30], which is then translated into a onedimensional distributed complex impedance $\left(Z_{c n t}\right)$ as detailed in Appendix A, Eqs. (A.1) - (A.2). The Drude-like surface conductivity $\left(\sigma_{c n t}\right)$ agrees well with the quantum mechanical dynamic conductivity model from microwave through 100 $\mathrm{THz}$ frequency range provided the CNTs are small radius, metallic, and single walled [41].

The full wave solver developed in our present study is capable of characterizing the scattering response of embedded CNTs for a wide frequency range, starting from $1 \mathrm{GHz}$ up to $10 \mathrm{THz}$. The sandwiched layer 2 can be as thick as $2 \mathrm{~mm}$. All three media can be assigned a broad range of electrical properties $\left(\varepsilon_{r i} / \mu_{r i} / \sigma_{i}\right)$. The interacting CNTs can be as far apart as $1 \mathrm{~cm}$ in lateral separation. The range of the parameter values considered for the CNT composite problem is summarized in Table I, which shows its true multiscale nature. In the absence of any embedded CNT, the fraction of the plane wave excitation that is transmitted from layer 1 into layer 2 experiences multiple reflections and transmissions at top and bottom interfaces, and eventually sets up an equivalent electric field in layer $2\left(\bar{E}_{2}^{i n c}(\bar{R})\right)$. When CNTs are present in layer $2, \bar{E}_{2}^{i n c}(\bar{R})$ will act as an excitation field and induce current $\left(\bar{I}_{c n t}\right)$ on the CNT surface, which in turn will produce scattering electric fields $\left(\bar{E}_{2}^{s}(\bar{R})\right)$. The boundary condition that relates the total tangential electric field to the induced axial current on the CNT is given below.

$$
\left[\bar{E}_{2}^{i n c}(\bar{R})+\bar{E}_{2}^{s}(\bar{R})\right]_{t a n}=Z_{c n t} \bar{I}_{c n t}
$$

One needs to solve Eq. (1) for every operating frequency to find the value of $\bar{I}_{c n t}$ that is required to compute the absorbed power spectrum of the embedded CNTs in the composite structure. We first compute the scattered electric field $\left(\bar{E}_{2}^{s}(\bar{R})\right)$ from the CNT surface, which is expressed as [28],

$$
\bar{E}_{2}^{s}(\bar{R})=-j \omega \mu_{2} \int_{l} \overline{\bar{G}}_{e}^{(22)}\left(\bar{R}, \bar{R}^{\prime}\right) \cdot \bar{I}_{c n t}\left(\bar{R}^{\prime}\right) d l
$$

where $\bar{R}$ is the observation vector (see Fig. 1) that locates the electric field scattering point on the CNT surface. $\bar{R}^{\prime}$ is the source vector, which locates the point on the CNT where the unknown axial current $\bar{I}_{c n t}\left(\bar{R}^{\prime}\right)$ is excited on an infinitesimal CNT length $d l$. The integration is computed over the contour length of the CNT $(l)$. The term $\bar{G}_{e}^{(22)}\left(\bar{R}, \bar{R}^{\prime}\right)$ is the spatial domain electric dyadic Green's function (DGF) of the third kind defined in layer 2, which will be discussed next.

\section{B. Spatial Domain Dyadic Green's Function (DGF)}

For a stratified three-layer medium with embedded radiating source, there exists a total of nine electric DGF of the third kind, represented as $\overline{\bar{G}}_{e}^{(p q)}\left(\bar{R}, \bar{R}^{\prime}\right)$ with $p, q=1,2,3$, where $p$ denotes observation layer and $q$ denotes source layer [29]. In our present study, we are interested in analyzing interactions among the buried CNTs in layer 2. So the source points $\left(\bar{R}^{\prime}\left(x^{\prime}, y^{\prime}, z^{\prime}\right)\right)$ and observation points $(\bar{R}(x, y, z))$, whether on the same CNT or on two different CNTs, are both located in layer $2(p=q=2)$ as shown in Fig. 1. Thus out of nine electric DGF, only $\bar{G}_{e}^{(22)}\left(\bar{R}, \bar{R}^{\prime}\right)$ is required to calculate scattering electric fields $\left(\bar{E}_{2}^{s}\right)$ in layer 2 as declared in (2). Following the method of scattering superposition, $\bar{G}_{e}^{(22)}\left(\bar{R}, \bar{R}^{\prime}\right)$ can be expressed in its generic form as given in [28], [29],

$$
\overline{\bar{G}}_{e}^{(22)}\left(\bar{R}, \bar{R}^{\prime}\right)=\overline{\bar{G}}_{e o}^{(2)}\left(\bar{R}, \bar{R}^{\prime}\right)+\overline{\bar{G}}_{e s}^{(22)}\left(\bar{R}, \bar{R}^{\prime}\right)
$$

where the first term on the right hand side denotes the free-space electrical DGF defined in a medium of the same constitutive constants as that of layer 2, and the second term defines the scattered DGF in layer 2. Considering the coordinate system and generic medium properties of Fig. 1, we derived the expression for $\bar{G}_{e}^{(22)}\left(\bar{R}, \bar{R}^{\prime}\right)$ as given below. However, for brevity the intermediate steps are skipped here, and can be followed in [28].

For $z>z^{\prime}$ in layer 2,

$$
\begin{aligned}
& \overline{\bar{G}}_{e}^{(22)}\left(\bar{R}, \bar{R}^{\prime}\right)=-\frac{1}{k_{2}^{2}} \hat{z} \hat{z} \delta\left(\bar{R}-\bar{R}^{\prime}\right)+\int_{0}^{\infty} d \lambda \sum_{n=0}^{\infty} \frac{j\left(\delta_{0}-2\right)}{4 \pi \lambda h_{2}} \\
& \left\{\frac{1}{\Gamma}\left[\bar{M}\left(+h_{2}\right)+\rho_{21} e^{-2 j h_{2} d} \bar{M}\left(-h_{2}\right)\right]\left[\rho_{23} \bar{M}^{\prime}\left(+h_{2}\right)+\bar{M}^{\prime}\left(-h_{2}\right)\right]\right. \\
& \left.+\frac{1}{\Gamma^{\prime}}\left[\bar{N}\left(+h_{2}\right)+\rho_{21}^{\prime} e^{-2 j h_{2} d} \bar{N}\left(-h_{2}\right)\right]\left[\rho_{23}^{\prime} \bar{N}^{\prime}\left(+h_{2}\right)+\bar{N}^{\prime}\left(-h_{2}\right)\right]\right\}
\end{aligned}
$$

and for $z<z^{\prime}$ in layer 2 ,

$$
\begin{aligned}
& \overline{\bar{G}}_{e}^{(22)}\left(\bar{R}, \bar{R}^{\prime}\right)=-\frac{1}{k_{2}^{2}} \hat{z} \hat{z} \delta\left(\bar{R}-\bar{R}^{\prime}\right)+\int_{0}^{\infty} d \lambda \sum_{n=0}^{\infty} \frac{j\left(\delta_{0}-2\right)}{4 \pi \lambda h_{2}} \\
& \left\{\frac{1}{\Gamma}\left[\rho_{23} \bar{M}\left(+h_{2}\right)+\bar{M}\left(-h_{2}\right)\right]\left[\bar{M}^{\prime}\left(+h_{2}\right)+\rho_{21} e^{-2 j h_{2} d} \bar{M}^{\prime}\left(-h_{2}\right)\right]\right. \\
& \left.+\frac{1}{\Gamma^{\prime}}\left[\rho_{23}^{\prime} \bar{N}\left(+h_{2}\right)+\bar{N}\left(-h_{2}\right)\right]\left[\bar{N}^{\prime}\left(+h_{2}\right)+\rho_{21}^{\prime} e^{-2 j h_{2} d} \bar{N}^{\prime}\left(-h_{2}\right)\right]\right\}
\end{aligned}
$$

The parameters and functions in (4)-(5) are defined as follows. $\lambda$ is the lateral wave vector in all three layers and also the Fourier-Bessel integral variable, and $h_{i}$ is the $z$ directed 
wave vector for the $i$ th layer $(i=1,2,3)$, which defines the radiation condition,

$$
h_{i}=\left\{\begin{array}{cc}
\sqrt{k_{i}^{2}-\lambda^{2}} & \text { for } \lambda \leq k_{i} \\
\mp j \sqrt{\lambda^{2}-k_{i}^{2}} & \text { for } \lambda>k_{i}
\end{array}\right.
$$

where the - sign and + sign in front of the second condition in (6) must be chosen appropriately to identify poles in the top or bottom Riemann sheet, respectively. Condition (6), (if - sign) ensures that the Sommerfeld radiation conditions are obeyed [32]. $\bar{M}$ and $\bar{N}$ are the cylindrical vector wave functions, which are solutions to the wave equation in layer 2, describing the TE mode and TM mode, respectively, and are expressed as [29],

$$
\begin{aligned}
& \bar{M}\left(h_{2}\right)=\bar{M}_{e_{n \lambda}}\left(h_{2}\right)=\nabla \times\left[J_{n}(\lambda r){ }_{\sin }^{\cos } n \phi e^{-j h_{2} z}\right] \\
& =\left[\mp \frac{n J_{n}(\lambda r)}{r} \sin _{\cos } n \phi \hat{r}-\frac{\partial J_{n}(\lambda r)}{\partial r} \sin _{\cos }^{\cos } n \phi \hat{\phi}\right] e^{-j h_{2} z} \\
& \bar{N}\left(h_{2}\right)=\bar{N}_{e_{o n \lambda}}\left(h_{2}\right)=\frac{1}{k_{2}} \nabla \times \bar{M}_{e_{o n \lambda}}\left(h_{2}\right) \\
& =\frac{1}{k_{2}}\left[-j h_{2} \frac{\partial J_{n}(\lambda r)}{\partial r}{ }_{\sin }^{\cos } n \phi \hat{r} \pm \frac{j h_{2} n}{r} J_{n}(\lambda r) \cos _{\sin }^{\sin } n \phi \hat{\phi}\right. \\
& \left.+\lambda^{2} J_{n}(\lambda r){ }_{\sin }^{\cos } n \phi \hat{z}\right] e^{-j h_{2} z}
\end{aligned}
$$

where the subscript $e$ denotes the even mode, and $o$ denotes the odd mode. $J_{n}$ is the Bessel function of order $n$. The primed functions in (4)-(5), $\bar{M}^{\prime}$ and $\bar{N}^{\prime}$, are the excitation coefficients defined with respect to the source coordinate $\left(r^{\prime}, \phi^{\prime}, z^{\prime}\right)$ pertaining to $\bar{R}^{\prime} \cdot \bar{M}^{\prime}\left(+h_{2}\right)$ and $\bar{N}^{\prime}\left(+h_{2}\right)$ represent wavelets travelling downward for the TE and TM modes, respectively. Similarly, $\bar{M}^{\prime}\left(-h_{2}\right)$ and $\bar{N}^{\prime}\left(-h_{2}\right)$ represent wavelets travelling upward for the TE and TM modes, respectively. $\delta_{0}=1$ for the null mode $(n=0)$, and $\delta_{0}=0$ for other modes $(n \neq 0)$. For waves travelling from layer- $i$ towards layer- $j$, their Fresnel's coefficients of reflection for electric fields at the interface for TE modes and TM modes are given respectively as,

$$
\rho_{i j}=\frac{\mu_{j} h_{i}-\mu_{i} h_{j}}{\mu_{j} h_{i}+\mu_{i} h_{j}} ; \rho_{i j}^{\prime}=\frac{k_{j}^{2} \mu_{i} h_{i}-k_{i}^{2} \mu_{j} h_{j}}{k_{j}^{2} \mu_{i} h_{i}+k_{i}^{2} \mu_{j} h_{j}} .
$$

Finally, the expressions for the terms $\frac{1}{\Gamma}$ and $\frac{1}{\Gamma^{\prime}}$ are given by [29],

$$
\begin{aligned}
& \frac{1}{\Gamma}=\left(1-\rho_{21} \rho_{23} e^{-2 j h_{2} d}\right)^{-1} \\
& \frac{1}{\Gamma^{\prime}}=\left(1-\rho_{21}^{\prime} \rho_{23}^{\prime} e^{-2 j h_{2} d}\right)^{-1}
\end{aligned}
$$

Substituting the expressions of (7)-(10) into (4)-(5) will lead to the final expression for $\overline{\bar{G}}_{e}^{(22)}$ in layer 2 for $z \gtrless z^{\prime}$.

For any arbitrary current distribution with $x y z$ variation there are nine associated components of $\overline{\bar{G}}_{e}^{(22)}$ present in the EFIE as shown below,

$$
\left[\begin{array}{l}
E_{2 x}^{s} \hat{x} \\
E_{2 y}^{s} \hat{y} \\
E_{2 z}^{s} \hat{z}
\end{array}\right]=-j \omega \mu_{2} \int_{l}\left[\begin{array}{lll}
G_{x x} \hat{x} \hat{x} & G_{x y} \hat{x} \hat{y} & G_{x z} \hat{x} \hat{z} \\
G_{y x} \hat{y} \hat{x} & G_{y y} \hat{y} \hat{y} & G_{y z} \hat{y} \hat{z} \\
G_{z x} \hat{z} \hat{x} & G_{z y} \hat{z} \hat{y} & G_{z z} \hat{z} \hat{z}
\end{array}\right] \cdot\left[\begin{array}{l}
J_{2 x} \hat{x} \\
J_{2 y} \hat{y} \\
J_{2 z} \hat{z}
\end{array}\right] d l
$$

Presently, we are interested in studying horizontal (aligned in $x$-axis) and vertical (aligned in $z$-axis) embedded CNTs as

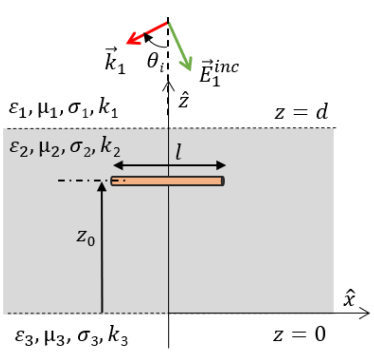

(a)

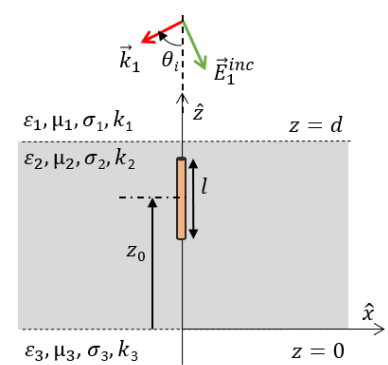

(b)
Fig. 2: (a) A horizontal CNT in layer 2 aligned parallel to the $x$-axis, and (b) a vertical CNT aligned parallel to the $z$-axis illuminated by a plane wave excitation polarized in $x z$-plane.

shown in Fig. 2. We choose the $x z$ plane as the plane of incidence for $\left(\bar{E}_{1}^{i n c}\right)$ that maximizes the field coupling to the CNTs in Fig. 2. Based on the thin wire approximation, the $x$ directed horizontal CNT in Fig. 2(a) couples only to the $E_{2 x}^{\text {inc }}$ component of the excitation field. Other components of $E_{2}^{i n c}$ have no effect on the $x$ directed horizontal CNT, and so do not contribute to the net electric field on the CNT surface, which then simply reduces to,

$$
E_{2 x}^{s}(\bar{R})=-j \omega \mu_{2} \int_{l} G_{x x}\left(\bar{R}, \bar{R}^{\prime}\right) J_{2 x}\left(\bar{R}^{\prime}\right) d l
$$

Similarly, the $z$ directed vertical CNT in Fig. 2(b) couples only to the $E_{2 z}^{i n c}$ component of the incident excitation and thus the net electric field on the $z$ directed CNT surface reduces to,

$$
E_{2 z}^{s}(\bar{R})=-j \omega \mu_{2} \int_{l} G_{z z}\left(\bar{R}, \bar{R}^{\prime}\right) J_{2 z}\left(\bar{R}^{\prime}\right) d l
$$

To evaluate the scattered electric fields we first need to evaluate the DGFs present in integrals (12) and (13). For planar stratified media, the DGFs are laterally invariant and thus while deriving $G_{x x}\left(\bar{R}, \bar{R}^{\prime}\right)$ and $G_{z z}\left(\bar{R}, \bar{R}^{\prime}\right)$ in layer 2 we consider the source point located at $\bar{R}^{\prime}\left(x^{\prime}=0, y^{\prime}=0, z^{\prime}\right)$, and choose an arbitrary observation point $\bar{R}(x, y, z)$. The expressions are as given below,

$$
\begin{aligned}
& G_{x x}\left(\bar{R}, \bar{R}^{\prime}\right)=\left[\bar{G}_{e}^{(22)}\left(\bar{R}, \bar{R}^{\prime}\right) \cdot \hat{x}\right]_{\left(0,0, z^{\prime}\right)} \cdot \hat{x} \\
& =\frac{-j}{4 \pi} \int_{0}^{\infty} d \lambda\left\{\lambda J_{0}(\lambda r) \sin ^{2}(\phi)+\frac{J_{1}(\lambda r)}{r} \cos (2 \phi)\right\} \widetilde{G}_{x x}^{T E} \\
& +\frac{j}{4 \pi k_{2}^{2}} \int_{0}^{\infty} d \lambda\left\{\lambda J_{0}(\lambda r) \cos ^{2}(\phi)-\frac{J_{1}(\lambda r)}{r} \cos (2 \phi)\right\} \widetilde{G}_{x x}^{T M}
\end{aligned}
$$

where $\hat{x}=\hat{r} \cos (\phi)-\hat{\phi} \sin (\phi)$ and,

$$
\begin{aligned}
& \widetilde{G}_{x x}^{T E}=\left(\frac{1}{h_{2} \Gamma}\right)\left\{\rho_{23} e^{-j h_{2}\left|z+z^{\prime}\right|}+e^{-j h_{2}\left|z-z^{\prime}\right|}\right. \\
& \left.+\rho_{21} e^{-2 j h_{2} d}\left(\rho_{23} e^{+j h_{2}\left|z-z^{\prime}\right|}+e^{+j h_{2}\left|z+z^{\prime}\right|}\right)\right\} \\
& \widetilde{G}_{x x}^{T M}=\left(\frac{h_{2}}{\Gamma^{\prime}}\right)\left\{\rho_{23}^{\prime} e^{-j h_{2}\left|z+z^{\prime}\right|}-e^{-j h_{2}\left|z-z^{\prime}\right|}\right. \\
& \left.-\rho_{21}^{\prime} e^{-2 j h_{2} d}\left(\rho_{23}^{\prime} e^{+j h_{2}\left|z-z^{\prime}\right|}-e^{+j h_{2}\left|z+z^{\prime}\right|}\right)\right\}
\end{aligned}
$$


Similarly,

$$
\begin{aligned}
& G_{z z}\left(\bar{R}, \bar{R}^{\prime}\right)=\left[\overline{\bar{G}}_{e}^{(22)}\left(\bar{R}, \bar{R}^{\prime}\right) \cdot \hat{z}\right]_{\left(0,0, z^{\prime}\right)} \cdot \hat{z} \\
& =-\frac{1}{k_{2}^{2}} \delta\left(\bar{R}-\bar{R}^{\prime}\right)-\frac{j}{4 \pi k_{2}^{2}} \int_{0}^{\infty} d \lambda\left\{\lambda^{3} J_{0}(\lambda r)\right\} \widetilde{G}_{z z}^{T M}
\end{aligned}
$$

where

$$
\begin{aligned}
\widetilde{G}_{z z}^{T M}= & \left(\frac{1}{h_{2} \Gamma^{\prime}}\right)\left\{\rho_{23}^{\prime} e^{-j h_{2}\left|z+z^{\prime}\right|}+e^{-j h_{2}\left|z-z^{\prime}\right|}\right. \\
& \left.+\rho_{21}^{\prime} e^{-2 j h_{2} d}\left(\rho_{23}^{\prime} e^{+j h_{2}\left|z-z^{\prime}\right|}+e^{+j h_{2}\left|z+z^{\prime}\right|}\right)\right\}
\end{aligned}
$$

The DGFs of (14) and (16) incorporate semi-infinite complex Sommerfeld integrals (SI) [32], which have no direct analytical solutions. The next sub-section discusses a semi-analytic approach in conjunction with special numerical treatments for efficient SI evaluation.

\section{Evaluation of Sommerfeld Integrals (SI) with Semi-analytic Approach} [32],

The generic form of a Sommerfeld integral is given below

$$
G\left(r, z, z^{\prime}\right)=\int_{0}^{\infty}\left\{J_{n}(\lambda r) \lambda^{n+1}\right\} \widetilde{G}\left(\lambda ; z, z^{\prime}\right) d \lambda
$$

which connects the spatial domain Green's function $G\left(r, z, z^{\prime}\right)$, to its spectral domain counterpart $\widetilde{G}\left(\lambda ; z, z^{\prime}\right)$. The spectral domain Green's function, $\widetilde{G}\left(\lambda ; z, z^{\prime}\right)$, also known as the layer/medium function, can take specific algebraic forms depending on the precise nature of the problem, such as in (15) and (17). The orders of Bessel function that survive the derivation from (3) - (17), are $n=0,1,2 . J_{2}(\lambda r)$, can be reduced to the lowest order Bessel functions $J_{0}(\lambda r)$ and $J_{1}(\lambda r)$ by using the recurrence relationship [42].

$$
J_{2}(\lambda r)=\frac{2}{\lambda r} J_{1}(\lambda r)-J_{0}(\lambda r)
$$

The fast and accurate evaluation of the Sommerfeld type integral is a classical problem that still draws a lot of attention from the computational EM community. Several computational techniques have emerged from decades of rigorous research to solve these semi-infinite integrals with competing performances in solution accuracy and computational speed [34], [36], [37], [43]-[54]. There exists no such single optimum integration strategy that works well for all types of Green's function problems. The solution strategy has to be adaptive following the nature of the particular problem in hand [55]. Especially when dealing with a multiscale multilayer problem with a wide frequency range, it becomes necessary to consider several factors that impact the behavior of the spectral domain Green's function $\widetilde{G}\left(\lambda ; z, z^{\prime}\right)$.

While evaluating SI on the complex $(\lambda)$ plane, severe numerical problems occur when source and observation point lie on the same plane. As $\left|z-z^{\prime}\right| \rightarrow 0$, the numerical integration begins to accumulate round off errors due to severe oscillatory behavior of the integrand induced by the Bessel functions [34], [48], [52].

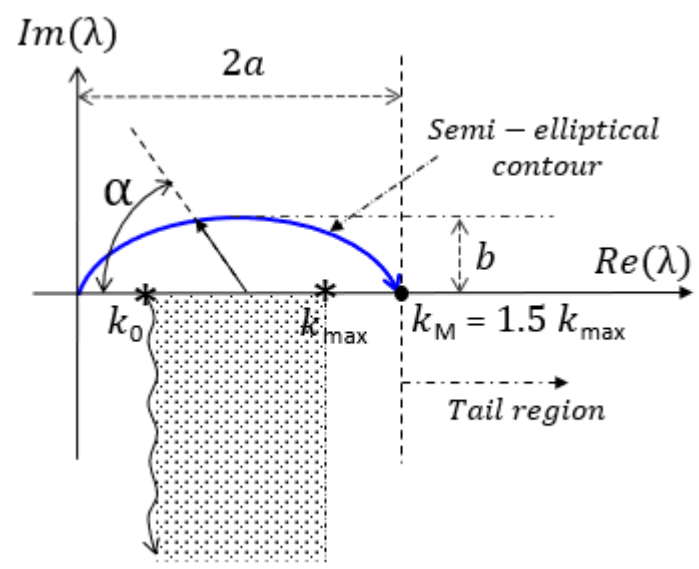

Fig. 3: Semi-analytical approach for Sommerfeld integral evaluation. The semi-elliptic contour deformation skips the region containing the integrand singularities lying in the range $k_{0} \leq \lambda \leq k_{\max }$. The tail part of the Sommerfeld integral $\left(k_{M} \leq \lambda \leq \infty\right)$ is evaluated in closed form.

If $\left|z-z^{\prime}\right| \not \supset 0$, and the integration path stays on the proper Riemann sheet, then the overall integrand may have a fast or slow convergence depending on the distribution of the exponential terms and the lateral separation of source and observation point. In this case, the integration along the semiinfinite $\mathcal{R} e(\lambda)$ axis still remains challenging, particularly when singularities of the integrand lie on or close to the $\operatorname{Re}(\lambda)$ axis, such as the case of low-loss layered structures [34], [48], [52].

There are finite numbers of surface wave poles (SWPs) and infinite numbers of leaky wave poles (LWPs) associated with $\widetilde{G}\left(\lambda ; z, z^{\prime}\right)$ [56]. The number of contributing poles increases with the electrical thickness of the embedding layer or with increasing operating frequency [35], [53], [56]. The pole locations depend on the constitutive parameter values, such as the material properties $\left\{\varepsilon_{i}, \mu_{i}, \sigma_{i}\right\}$, and layer thickness $(d)$ [35], [53], [56]. In order to integrate the SI on the $\mathcal{R} e(\lambda)$ axis, maintaining a high numerical accuracy from near field to far field calculations, one needs to identify all contributing pole locations accurately and evaluate their residue effects [52]-[54]. This is very complex iterative time consuming task that will hinder the goal of fast computation of the MoM matrix especially when the structure is lossy, multiscale, and multilayer.

Fortunately, these poles are dispersed in the shaded strip region, as shown in Fig. 3, confined by the branch points and branch cuts in the lower half plane (considering all media are right handed). To obviate the need of pole extraction and residue calculations, we chose to lift the contour of integration over the pole region in the first quadrant of the complex $(\lambda)$ plane and split the SI into two parts following [36], [49]:

$$
\begin{aligned}
& \int_{0}^{\infty}\left\{J_{n}(\lambda r) \lambda^{n+1}\right\} \widetilde{G}\left(\lambda ; z, z^{\prime}\right) d \lambda \\
& =\int_{0}^{k_{M}}\{\cdots\} \widetilde{G}\left(\lambda ; z, z^{\prime}\right) d \lambda+\int_{k_{M}}^{\infty}\{\cdots\} \widetilde{G}\left(\lambda ; z, z^{\prime}\right) d \lambda .
\end{aligned}
$$

The first finite integral on the right hand side from 0 to $k_{M}$, (with $k_{M} \geq \max \left\{\mathcal{R} e\left(k_{i=1,2,3}\right)\right\}$ ), is computed following a deformed contour (see Fig. 3) in the upper half plane, 
which avoids all branch points and singularities lying in the fourth quadrant of the complex $(\lambda)$ plane. Different choices of contour can be found in the literature, such as semi-circle, semi-ellipse, or triangle, among which we chose the semielliptical contour for its simple parametric representation and independent control over its major and minor axis [34], [36], [48]. The details of this contour deformation technique will be discussed at the end of this section.

The remaining semi-infinite integral from $k_{M}$ to $\infty$, commonly known as the Sommerfeld integral tail, is free from singularities thus can be integrated on the $\mathcal{R} e(\lambda)$ path. However, the numerical integration of the SI tail defined by oscillatory and slowly varying kernels is a time consuming task. Different algorithms have been proposed in the literature to efficiently calculate SI tails, such as weighted averages [51], double exponential (DE) [52], matrix pencil method (MPM) [44], multilevel DCIM [46], and modified fast Hankel transform method (MFHT) [50] etc. The pros and cons of these methods and their applicability have been nicely summarized [37]. However, expressing the SI tails in exact closed form can certainly outperform the above methods in computation time, especially when a $\mathrm{GHz}$ to $\mathrm{THz}$ range of operation is being considered with large lateral separations.

In this work we propose a new formulation to compute SI tails in exact closed form, which is inspired by the works of [34], [44], [47]-[49], but different in many aspects. We chose a sufficiently large suitable value of $k_{M}>\max \left\{\mathcal{R} e\left(k_{i=1,2,3}\right)\right\}$, such that when $\lambda \geq k_{M}$, we can express $\widetilde{G}\left(\lambda ; z, z^{\prime}\right)$ in an appropriate asymptotic form under the following conditions,

$$
\lim _{\lambda \rightarrow \infty}\left\{\begin{array}{c}
h_{1} \approx h_{3} \approx h_{2}=-j \sqrt{\lambda^{2}-k_{2}^{2}} \\
\rho_{i j} \approx \varrho_{i j}=\frac{\mu_{j}-\mu_{i}}{\mu_{j}+\mu_{i}} ; \rho_{i j}^{\prime} \approx \varrho_{i j}^{\prime}=\frac{k_{j}^{2} \mu_{i}-k_{i}^{2} \mu_{j}}{k_{j}^{2} \mu_{i}+k_{i}^{2} \mu_{j}} \\
\widetilde{G}\left(\lambda ; z, z^{\prime}\right) \asymp \widetilde{\mathcal{G}}\left(\lambda \geq k_{M} ; z, z^{\prime}\right)
\end{array}\right.
$$

where $\varrho_{i j}$ and $\varrho_{i j}^{\prime}$ become independent of the integration variable $\lambda$ and are indeed the low-frequency approximation of the reflection coefficients. The first condition of (21) leads to more accurate asymptotic approximation for $\widetilde{G}\left(\lambda ; z, z^{\prime}\right)$, compared to what was reported in [48]. In [47], the authors used a similar approximation but neglected the entire contour integration contribution, so that their approach was restricted only to the low frequency regime $(\omega \rightarrow 0)$. In contrast, our approach is full-wave and works accurately from low frequency through tens of $\mathrm{THz}$. Next, we focus on finding suitable closed form expressions for the SI tails. Following the idea of [49] and referring to Fig. 3, we express an alternate format for the SI tail, which is the second integral appearing on the right hand side of (20), as follows:

$$
\begin{aligned}
& \int_{k_{M}}^{\infty}\{\cdots\} \widetilde{G}\left(\lambda ; z, z^{\prime}\right) d \lambda \\
& \approx \int_{0}^{\infty}\{\cdots\} \widetilde{\mathcal{G}}\left(\lambda \geq k_{M} ; z, z^{\prime}\right) d \lambda \\
& \quad-\int_{0}^{k_{M}}\{\cdots\} \widetilde{\mathcal{G}}\left(\lambda \geq k_{M} ; z, z^{\prime}\right) d \lambda
\end{aligned}
$$

Substituting (22) into (20) and rearranging terms under similar integration limits one can obtain the approximate relationship for general numerical evaluation of Sommerfeld integrals appearing in various classes of multilayer media problems. This relationship reads:

$$
\begin{aligned}
& \int_{0}^{\infty}\{\cdots\} \widetilde{G}\left(\lambda ; z, z^{\prime}\right) d \lambda \\
& \approx \int_{0}^{k_{M}}\{\cdots\}\left[\widetilde{G}\left(\lambda ; z, z^{\prime}\right)-\widetilde{\mathcal{G}}\left(\lambda \geq k_{M} ; z, z^{\prime}\right)\right] d \lambda \\
& \quad+\int_{0}^{\infty}\{\cdots\} \widetilde{\mathcal{G}}\left(\lambda \geq k_{M} ; z, z^{\prime}\right) d \lambda
\end{aligned}
$$

The integrand subtraction appearing in the first integral on the right hand side is a key step towards efficient SI evaluation, as it serves two purposes:

1) After subtraction, the resultant integrand in the contour integral $\left[0, k_{M}\right]$ decays faster with $\lambda$, which accelerates the numerical integration process. Without subtraction, the original integrand for $\left[0, k_{M}\right]$ decays very slowly with $\lambda$, especially when the source and field points are close to each other $(\delta z \rightarrow 0)$ and close to an interface between two adjacent layers. In such cases, the exponential terms like $e^{-j h_{i} \delta z}$ present in $\widetilde{G}\left(\lambda ; z, z^{\prime}\right)$ approach 1 and do not decay with increasing $\lambda$ [48].

2) The SI tail integration limit is now modified to $[0, \infty)$, which eases the search for an exact closedform expression for the SI tail using the Fourier-Bessel transform relationships given in [57].

It is important to note that, unlike [43], and [44], the present method does not approximate the Bessel terms of (14) and (16) while searching for closed-form expressions for the SI tails. Asymptotic approximation of the Bessel/Hankel functions enhances inaccuracies for small separation between source and observation. The above techniques are unjustified for CNTs that have source points in $\mathrm{nm}$ scale proximity of observation points $\left(k_{0} r \approx 10^{-8}\right.$ at $\left.1 \mathrm{GHz}\right)$. Thus, in the present method, the asymptotic condition (21) is imposed only on the spectral domain Green's functions of (15) and (17). For $\lambda \geq k_{M}$, we first substitute (21) into (10) and expand it into power series as follows,

$$
\begin{aligned}
\frac{1}{\Gamma} & \approx\left(1-\varrho_{21} \varrho_{23} e^{-2 d \sqrt{\lambda^{2}-k_{2}^{2}}}\right)^{-1} \\
& \approx \sum_{n=0}^{\infty}\left(\varrho_{21} \varrho_{23} e^{-2 d \sqrt{\lambda^{2}-k_{2}^{2}}}\right)^{n} \\
\frac{1}{\Gamma^{\prime}} & \approx\left(1-\varrho_{21}^{\prime} \varrho_{23}^{\prime} e^{-2 d \sqrt{\lambda^{2}-k_{2}^{2}}}\right)^{-1} \\
& \approx \sum_{n=0}^{\infty}\left(\varrho_{21}^{\prime} \varrho_{23}^{\prime} e^{-2 d \sqrt{\lambda^{2}-k_{2}^{2}}}\right)^{n}
\end{aligned}
$$

The convergence of the above power series is guaranteed since the magnitude of the second term inside $(\cdots)^{-1}$ is always less than one. Now, inserting (24) and (21) back into (15) we get, 


$$
\begin{aligned}
& \widetilde{\mathcal{G}}_{x x}^{T E}=\frac{j}{\sqrt{\lambda^{2}-k_{2}^{2}}} \sum_{m=1}^{4} \sum_{n=0}^{\infty} b_{m n} e^{-a_{m n} \sqrt{\lambda^{2}-k_{2}^{2}}} \\
& \widetilde{\mathcal{G}}_{x x}^{T M}=-j \sqrt{\lambda^{2}-k_{2}^{2}} \sum_{m=1}^{4} \sum_{n=0}^{\infty} c_{m n} e^{-a_{m n} \sqrt{\lambda^{2}-k_{2}^{2}}}
\end{aligned}
$$

where,

$$
\begin{aligned}
a_{1 n} & =2 d(n-1)+z+z^{\prime}, & a_{2 n} & =2 d n-\left|z-z^{\prime}\right| \\
a_{3 n} & =2 d(n-1)+\left|z-z^{\prime}\right|, & a_{4 n} & =2 d n-z-z^{\prime} \\
b_{1 n} & =\varrho_{23}\left(\varrho_{21} \varrho_{23}\right)^{n-1}, & b_{2 n} & =\left(\varrho_{21} \varrho_{23}\right)^{n} \\
b_{3 n} & =\left(\varrho_{21} \varrho_{23}\right)^{n-1}, & b_{4 n} & =\varrho_{21}\left(\varrho_{21} \varrho_{23}\right)^{n-1} \\
c_{1 n} & =\varrho_{23}^{\prime}\left(\varrho_{21}^{\prime} \varrho_{23}^{\prime}\right)^{n-1}, & c_{2 n} & =-\left(\varrho_{21}^{\prime} \varrho_{23}^{\prime}\right)^{n} r \\
c_{3 n} & =-\left(\varrho_{21}^{\prime} \varrho_{23}^{\prime}\right)^{n-1}, & c_{4 n} & =\varrho_{21}^{\prime}\left(\varrho_{21}^{\prime} \varrho_{23}^{\prime}\right)^{n-1}
\end{aligned}
$$

Similarly, inserting (24b) and (21) into (17) we get,

$$
\widetilde{\mathcal{G}}_{z z}^{T M}=\frac{j}{\sqrt{\lambda^{2}-k_{2}^{2}}} \sum_{m=1}^{4} \sum_{n=0}^{\infty} d_{m n} e^{-a_{m n}} \sqrt{\lambda^{2}-k_{2}^{2}}
$$

where the $a_{m n}$ terms are the same as in (25), and

$$
d_{1 n}=c_{1 n}, d_{2 n}=-c_{2 n}, d_{3 n}=-c_{3 n}, d_{4 n}=c_{4 n}
$$

Now we substitute (25) into (14) and express it in the form of (23) as given below,

$$
\begin{aligned}
& G_{x x}\left(\bar{R}, \bar{R}^{\prime}\right) \approx\left(\left.G_{x x}^{T E}\right|_{\text {contour }}+\left.G_{x x}^{T E}\right|_{\text {closed }}\right) \\
&+\left(\left.G_{x x}^{T M}\right|_{\text {contour }}+\left.G_{x x}^{T M}\right|_{\text {closed }}\right) \\
&\left.G_{x x}^{T E}\right|_{\text {contour }}=-\frac{j}{4 \pi} \int_{0}^{k_{M}}\left\{\lambda J_{0}(\lambda r) \sin ^{2}(\phi)+\frac{J_{1}(\lambda r)}{r} \cos (2 \phi)\right\} \times\left[\widetilde{G}_{x x}^{T E}-\widetilde{\mathcal{G}}_{x x}^{T E}\right] d \lambda \\
&\left.G_{x x}^{T E}\right|_{\text {closed }}=-\frac{j}{4 \pi} \int_{0}^{\infty}\left\{\lambda J_{0}(\lambda r) \sin ^{2}(\phi)+\frac{J_{1}(\lambda r)}{r} \cos (2 \phi)\right\} \\
& \times \widetilde{\mathcal{G}}_{x x}^{T E} d \lambda \\
&\left.G_{x x}^{T M}\right|_{\text {contour }}=\frac{j}{4 \pi k_{2}^{2}} \int_{0}^{k_{M}}\left\{\lambda J_{0}(\lambda r) \cos ^{2}(\phi)-\frac{J_{1}(\lambda r)}{r} \cos (2 \phi)\right\} \\
& \times\left[\widetilde{G}_{x x}^{T M}-\widetilde{\mathcal{G}}_{x x}^{T M}\right] d \lambda \\
&\left.G_{x x}^{T M}\right|_{\text {closed }}=\frac{j}{4 \pi k_{2}^{2}} \int_{0}^{\infty}\left\{\lambda J_{0}(\lambda r) \cos ^{2}(\phi)-\frac{J_{1}(\lambda r)}{r} \cos (2 \phi)\right\} \\
& \times \widetilde{\mathcal{G}}_{x x}^{T M} d \lambda
\end{aligned}
$$

Similarly, by inserting (26) into (16) we get,

$$
\begin{aligned}
& G_{z z}\left(\bar{R}, \bar{R}^{\prime}\right) \approx-\frac{1}{k_{2}^{2}} \delta\left(\bar{R}-\bar{R}^{\prime}\right)+\left(\left.G_{z z}^{T M}\right|_{\text {contour }}+\left.G_{z z}^{T M}\right|_{\text {closed }}\right) \\
& \left.G_{z z}^{T M}\right|_{\text {contour }}=-\frac{j}{4 \pi k_{2}^{2}} \int_{0}^{k_{M}}\left\{\lambda^{3} J_{0}(\lambda r)\right\}\left[\widetilde{G}_{z z}^{T M}-\widetilde{\mathcal{G}}_{z z}^{T M}\right] d \lambda \\
& \left.G_{z z}^{T M}\right|_{\text {closed }}=-\frac{j}{4 \pi k_{2}^{2}} \int_{0}^{\infty}\left\{\lambda^{3} J_{0}(\lambda r)\right\} \widetilde{\mathcal{G}}_{z z}^{T M} d \lambda
\end{aligned}
$$

In (27) we find two finite and two infinite integrals, whereas in (28) there is one finite and one infinite integral. All these integrals include infinite series in their integrands coming from the $\widetilde{\mathcal{G}}$ terms. However, the infinite series can be truncated to a finite series without losing accuracy by judicially choosing a sufficiently large value of $n$. In our present study, we use $n=500$ to eliminate any cumulative approximation error in the MoM calculation. However, this is not an ultimate value of $n$ and can be optimized to reduce the SI tail computation time while maintaining required solution accuracy. As indicated by the subscripts, the finite integrals $\left.G_{x x}^{T E}\right|_{\text {contour }},\left.G_{x x}^{T M}\right|_{\text {contour }}$, and $\left.G_{z z}^{T M}\right|_{\text {contour }}$ are computed numerically by integrating over the semi-elliptical contour. In this case, each $\widetilde{\mathcal{G}}$ is computed first as a finite series up to $n$th terms and then subtracted from $\widetilde{G}$ giving the resultant spectral domain Green's function, which is then multiplied with Bessel terms followed by contour integration. For the infinite integrals, $\left.G_{x x}^{T E}\right|_{\text {closed }},\left.G_{x x}^{T M}\right|_{\text {closed }}$, and $\left.G_{z z}^{T M}\right|_{\text {closed }}$, fortunately no integration is needed as we find exact closed-form solutions from [57] using Fourier-Bessel transform relationships as listed in Appendix B. To do so, we move the summation operator in front of the integration. This step is valid since the summation coefficients $b_{m n}, c_{m n}$, and $d_{m n}$ are independent of the spectral variable $\lambda$. This step allow us to express $\left.G_{x x}^{T E}\right|_{\text {closed }}$ as a sum of exponential terms by using the Sommerfeld identity (B.1) and its derivative (B.2). Similarly, we can express $\left.G_{x x}^{T M}\right|_{\text {closed }}$ as a sum of exponential terms by using the identities (B.3) and (B.4). $\left.G_{z z}^{T M}\right|_{\text {closed }}$ is expressed as a sum of exponential terms by using (B.5).

As we now have the closed form solutions for the SI tails in (27) and (28), we finally turn our attention to evaluate the finite integrals over the semi-elliptic contour in the upper half of the complex $\lambda$ plane. As depicted in Fig. 3, we first transform the finite integrals from $\lambda:\left[0, k_{M}\right]$ space to $\alpha:[0, \pi]$ space by applying the following semi-elliptic relations,

$$
\left\{\begin{array}{c}
\lambda=a-a \cos \alpha+j b \sin \alpha \\
\frac{d \lambda}{d \alpha}=a \sin \alpha+j b \cos \alpha
\end{array}\right.
$$

where $a=\frac{k_{M}}{2}$ is the semi-major axis and $b$ is the semi-minor axis of the semi-elliptic contour. The value of $k_{M}$, or the major axis length, should be large enough to skip any singularities near the path of integration and also ensure the validity of the asymptotic condition (21). The minor axis height $b$ dictates the convergence of the integrands, which are highly oscillatory along the $\mathcal{R} e(\lambda)$ axis and exponential along the $\mathcal{I} m(\lambda)$ axis [52]. A large value of $b$ will cause both $J_{0,1}(\lambda r)$ terms to diverge. Reducing $b$ significantly can bring the contour down 
TABLE II: Controlling Minor Axis Height (b)

\begin{tabular}{|c|c|c|c|c|c|}
\hline \multirow{3}{*}{ Reference } & \multirow{3}{*}{ Choice of $b$} & \multicolumn{4}{|c|}{ Value of $b$} \\
\hline & & \multicolumn{2}{|c|}{$f=1 \mathrm{GHz}$} & \multicolumn{2}{|c|}{$f=10 \mathrm{THz}$} \\
\hline & & $r=0.61 \mathrm{~nm}$ & $r=1 \mathrm{~cm}$ & $r=0.61 \mathrm{~nm}$ & $r=1 \mathrm{~cm}$ \\
\hline [35], [36] & $b=\left\{\begin{array}{cc}k_{0} \times \min \left(1, \frac{1}{k_{0} r}\right) & \text { for } \quad r>\left|z-z^{\prime}\right| \\
k_{0} & \text { for } \quad r \leq\left|z-z^{\prime}\right|\end{array}\right.$ & 20.95 & 20.95 & $2.09 \times 10^{5}$ & $2.09 \times 10^{5}$ \\
\hline [34], [48] & $\begin{aligned} b= & 10^{-3} \times 1.2 \times \max \left\{\operatorname{Re} e\left(k_{i}\right)\right\} \\
& =10^{-3} \times 1.2 \times \mathcal{R} e\left(k_{2}\right)\end{aligned}$ & 0.098 & 0.098 & 795 & 795 \\
\hline This work & $b=\left\{\begin{array}{cc}20 \mathrm{~m}^{-1} & \text { for } \quad 1 \mathrm{GHz} \leq f \leq 2 \mathrm{THz} \\
10 \times \frac{f}{10^{12} \mathrm{~Hz}^{-1}} & \text { for } \quad 2 \mathrm{THz}<f \leq 10 \mathrm{THz}\end{array}\right.$ & 20 & 20 & 100 & 100 \\
\hline
\end{tabular}

Considering $\varepsilon_{r 2}=10, \mu_{r 2}=1, \sigma_{2}=1 \mathrm{~S} / \mathrm{m}$. Air for layer1 and layer3.

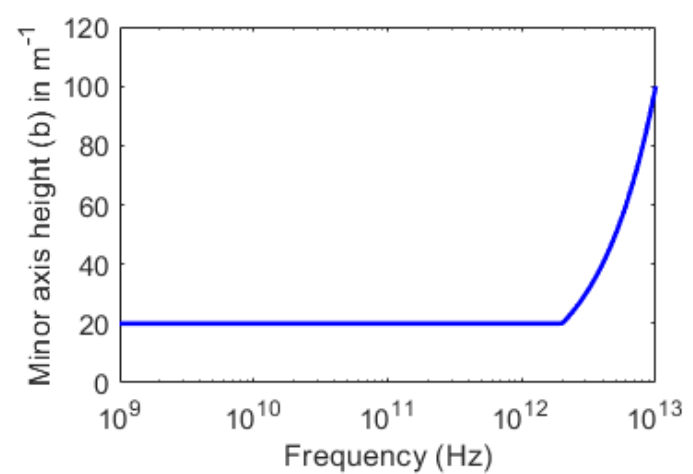

Fig. 4: Semi-elliptic contour minor axis height (b) with varying range of frequency.

to the vicinity of singularities lying close to, or on the $\mathcal{R} e(\lambda)$ axis [34], [52]. Thus an optimal choice of $b$ that minimizes the effect of the poles and the oscillations of the Bessel function should be somewhere in between these two extreme limits. A few studies can be found in this context that discuss how to control the semi-minor axis height and are given in Table II. However, none of these guidelines are fully compatible for a $\mathrm{GHz}$ to $\mathrm{THz}$ frequency range and $\mathrm{nm}$ to $\mathrm{cm}$ lateral range of operation. As shown in Table II, if we follow [35], [36], at 10 $\mathrm{THz}$ the value of $b$ will become too large and the integrand will diverge. If we follow [34], [48], at $1 \mathrm{GHz}$ the value of $b$ will become too small and the contour will come very close to $\operatorname{Re}(\lambda)$ axis, and at $10 \mathrm{THz}$ the value of $b$ will be large enough to cause the integrand to diverge. Thus, in our present study we propose a new adaptive contour height that is suitable for our multiscale scenario (see Table I). The contour height variation with frequency is shown in Fig. 4 and the relation is given in the last row in Table II. The contour maintains a constant low value of $b$ as long as the operating frequency lies below $2 \mathrm{THz}$. Further increase in frequency enhances the effects of singularities near the $\mathcal{R} e(\lambda)$ axis and it becomes necessary to push the contour further into the upper half plane. This is unavoidable because with increasing frequency layer 2 becomes electrically thick and more higher order poles pop up near $\operatorname{Re}(\lambda)$ axis. So eventually, $a$ as well as $b$ are increased to avoid the effects of singularities. This effect is more concerning when the source is close to an interface as it induces convergence problems. Our proposed choice of $b$ has been tested for all possible scenarios of operating frequency, physical length, and material properties given in Table I. The validation results will be shown in section III.

\section{MoM-ATW for Embedded CNTs}

The calculated scattered electric field value $\bar{E}_{2}^{s}(\bar{R})$ is inserted into the E-field boundary condition on the CNT surface (1) to solve the unknown current density flowing axially in the ATW CNT $\left(\bar{I}_{c n t}\right)$. We first assume that the individual ATW CNT structure is subdivided into multiple number of connected small segments $(S)$, and (1) is solved for each of these segments. The unknown $\bar{I}_{c n t}$ thus can be approximately expressed as a linear weighted summation of $N=S-1$ overlapped triangular basis functions:

$$
\bar{I}_{c n t} \approx \sum_{n=1}^{N} I_{n} \bar{f}_{n}(\bar{R})
$$

where $I_{n}$ is the unknown weighting current coefficient for the $n$th triangular basis $\bar{f}_{n}$ that expands over $n$th and $(n+1)$ th segment [31]. To satisfy the ATW condition with reasonable accuracy, the discretization should be optimized. At least 20 segments per wavelength is desired, but individual segment length should not fall below twice the diameter of the CNT [13], [16]. This sets an upper and lower limit on the choice of segment number $(\mathrm{S})$,

$$
\frac{20 l}{\lambda}<S<\frac{l}{4 r_{c n t}}
$$

Substituting (30) into (1) results in a matrix equation as given below that needs to be solved for each operating frequency [31],

$$
[Z]_{N \times N}[I]_{N \times 1}=[V]_{N \times 1}
$$

On the right hand side, $V=\left[\bar{E}_{2}^{i n c}(\bar{R})\right]_{t a n}$ is the tangential E-field component of the incident excitation that couples to the CNT. On the left hand side, $Z$ is the resultant complex impedance matrix, also known as the MoM impedance matrix. To find $Z$, we have followed the procedure described in [31]. We first set the right hand side of (1) to zero, which gives us the solution of the impedance matrix of a perfect 
electric conductor (PEC) wire. Next, we add the distributed line impedance value of CNT $\left(Z_{c n t}\right)$ along the diagonal elements the PEC impedance matrix calculated in the previous step. Imposing the right impedance condition along the thin wire structure is the key step that differentiates the CNT electromagnetic response from that of a similar PEC wire. The total axial current $\left(\bar{I}_{c n t}\right)$ flowing in the CNT is determined by solving the matrix equation (32). Once the current is solved, we can finally calculate the total extinction power $\left(P_{\text {ext }}\right)$, absorbed power $\left(P_{a b s}\right)$, and scattered power $\left(P_{\text {scat }}\right)$ for the embedded CNTs following the given relations [13],

$$
\begin{aligned}
& P_{\text {ext }}=0.5 \mathcal{R} e\left(\int_{0}^{l} \bar{I}_{c n t}^{\dagger} \cdot \bar{E}_{2}^{i n c}(\bar{R}) d l\right) \\
& P_{a b s}=0.5 \int_{0}^{l}\left|\bar{I}_{c n t}\right|^{2} \mathcal{R} e\left(Z_{c n t}\right) d l \\
& P_{\text {scat }}=P_{\text {ext }}-P_{a b s}
\end{aligned}
$$

where the ${ }^{\dagger}$ notation refers to conjugate transpose. For PEC and conventional metals such as gold, silver, or aluminum, $P_{\text {scat }}$ dominates over $P_{a b s}$, whereas conductors with complex conductivity such as the CNTs show higher values of $P_{a b s}$ compared to $P_{\text {scat }}$. The resonant peaks found in the absorption power spectrum have various applications in CNT-based nanosensing platforms.

Filling the impedance matrix $[Z]_{N \times N}$ in (32) is a time consuming task. To give an idea, the matrix size or the number of bases $(N)$ may range from tens (for a single CNT) to several thousand (for a cluster of CNTs). For more accurate numerical integration, we have subdivided each basis into 64 LegendreGauss quadrature points. Thus to calculate $[Z]_{N \times N}$ for a single operating frequency, one needs to compute the source observation interactions for $64 \times N \times 64 \times N$ times. However, considering the source observation reciprocity, and lateral invariance properties of the Green's function of multilayered structures, we can reduce the burden of repetitive calculations. To accelerate the filling of $[Z]_{N \times N}$ we have adapted different interpolation and look-up-table (LUT) strategies. In general, if all other constitutive parameter values remain constant, then a spatial domain Green's function $G\left(r, z, z^{\prime}\right)$ needs a three-dimensional (3D) LUT for varying lateral separation between source and observation point $(r)$, observation height $(z)$, and source height $\left(z^{\prime}\right)$. For a horizontal CNT, $z$ and $z^{\prime}$ are fixed, and thus for $G_{x x}$ a one-dimensional (1D) LUT with varying $r$ is sufficient. However, for a vertical CNT only $r$ is fixed, thus for $G_{z z}$ a two-dimensional (2D) LUT with varying $z$ and $z^{\prime}$ is required. In our present study, a LUT is built only for the contour integration part of the SI, which is otherwise a laborious task especially at $\mathrm{THz}$ frequencies. Spline interpolation is used to populate the contour integration LUT for intermediate locations between the node points. The closed form expressions for the SI tail are easy to compute and require minimal time, thus no separate LUT were used for them. For the self terms (diagonal entries in $[Z]$ matrix) and terms adjacent to them, which inherently have a large order of magnitude, their closed parts are filled by exhaustive calculations. Source and observation bases that are at least a segment apart are filled by a $5 \times 5$ spline interpolation for each segment consisting of $32 \times 32$ Legendre-Gauss quadrature points. This strategy ensures the high accuracy of the fullwave solver and at the same time maintains a fast computing speed. Details of the computational speed and accuracy of the solution compared to commercial solvers are discussed in the following sections.

\section{Electric Field EVAluation AND Validation}

We first demonstrate the validity of the Green's function formulated in (27) and (28). We consider a $x$-directed HED (horizontal electric dipole) and $z$-directed VED (vertical electric dipole) of unit magnitude current embedded in a lossy dielectric slab as shown schematically in Fig. 5a and Fig. $6 \mathrm{a}$, respectively. The dielectric slab is $d=2 \mathrm{~mm}$ thick and is backed by air $\left(\varepsilon_{r 1,3}=1, \mu_{r 1,3}=1, \sigma_{1,3}=0 \mathrm{~S} / \mathrm{m}\right)$ on either side. The electric dipoles are placed $\delta z=0.25 \mathrm{~mm}$ below the top interface. The dominant electric field $E_{2 x}$ for $x$-directed HED is computed directly following (12), which is nothing but $G_{x x}$ multiplied with a factor of $\left(-j \omega \mu_{2}\right)$. Similarly, the dominant electric field $E_{2 z}$ for $z$-directed VED is computed directly following (13), which is actually $G_{z z}$ multiplied with a factor of $\left(-j \omega \mu_{2}\right)$. The dominant field components are shown specifically as they will be required to solve the embedded $x$-directed and $z$-directed CNTs of Fig. 2. The in-house field calculations are validated against the results generated by the FEKO planar multilayer substrate solver which also uses a multilayer Green's function formulation. We validate the field computation rigorously for the wide frequency range from $1 \mathrm{GHz}$ to $10 \mathrm{THz}$ and parameters as given in Table I. However, for brevity we select $f=1 \mathrm{THz}$ as an intermediate frequency value and select two different sets of slab dielectric properties to present the validation study as shown in Fig. 5b-5c and Fig. 6b-6c. The electric fields produced by HED in Fig. 5b and VED in Fig. $6 \mathrm{~b}$ consider a dielectric slab having electrical properties close to that of lossless air $\left(\varepsilon_{r 2}=1.1, \mu_{r 2}=1, \sigma_{2}=10^{-9} \mathrm{~S} / \mathrm{m}\right)$. In Fig. 5c and Fig. $6 \mathrm{c}$, the slab is assigned high values of permittivity and conductivity $\left(\varepsilon_{r 2}=20, \mu_{r 2}=1, \sigma_{2}=20 \mathrm{~S} / \mathrm{m}\right)$. The E-fields were computed at the source height $\left(z=z^{\prime}\right)$ for the sourceobservation lateral separation starting from $r_{c n t}=0.61 \mathrm{~nm}$ to $1 \mathrm{~cm}$. For a clean comparison, the computed real and imaginary part of the electric fields are plotted separately along with the corresponding FEKO results. The relative percentage error/difference between the in-house field calculation and FEKO result is calculated as follows,

$$
\text { Relative error }=\frac{E-E_{F E K O}}{E_{F E K O}} \times 100 \%
$$

The relative error remains less than $1 \%(<-2 \mathrm{~dB})$ (grey dashed line) throughout the lateral range. The validation study clearly shows that the in-house DGF implementation is robust, accurate and suitable for the multiscale composite environment, which includes extremely thick layers, variable media properties, and THz-range operating frequency.

\section{Absorption Power Spectrum of Embedded CNTs}

In this section, we perform full-wave analysis of both horizontal and vertical embedded CNTs in a lossy dielectric slab. 


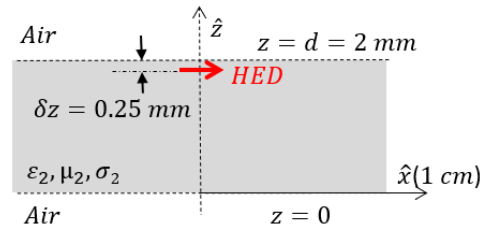

(a)
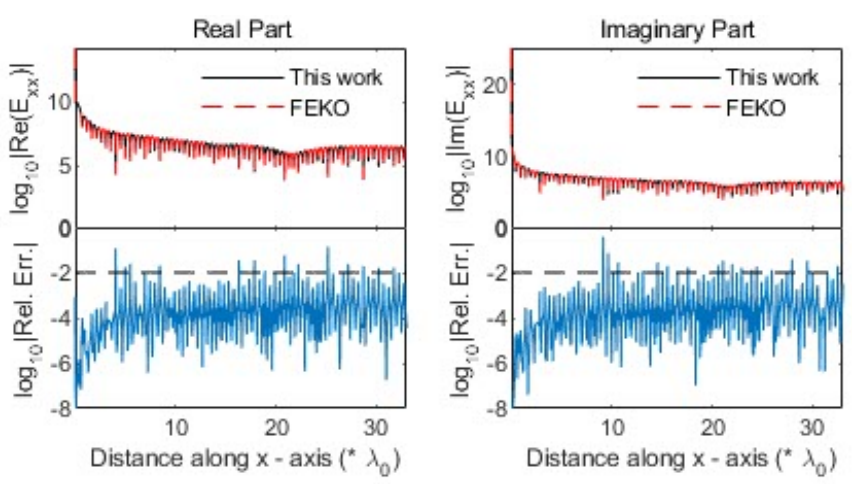

(b)
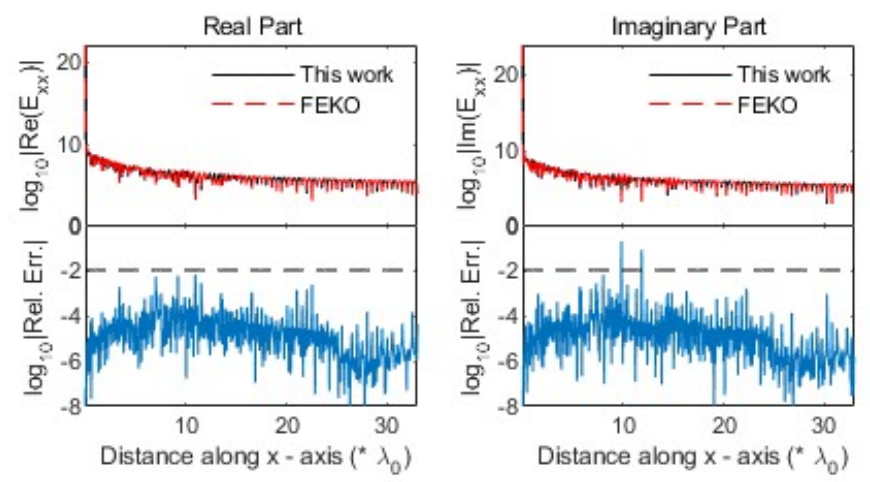

(c)

Fig. 5: (a) Schematic diagram of an $x$-directed electric dipole placed inside a $d=2 \mathrm{~mm}$ thick semi-infinite lossy dielectric slab at a depth of $\delta z=0.25 \mathrm{~mm}$ from the top interface. The top and bottom layers are considered as air. The $x$-component of the electric field is computed at the source height at $f=1 \mathrm{THz}\left(\lambda_{0}=3 \times 10^{-4} \mathrm{~m}\right)$ for a lateral range of $x=0.61 \mathrm{~nm}$ to $1 \mathrm{~cm}$, for the following two different lossy dielectric cases, (b) $\varepsilon_{r 2}=1.1, \mu_{r 2}=1, \sigma_{2}=10^{-9} \mathrm{~S} / \mathrm{m}$, (c) $\varepsilon_{r 2}=20, \mu_{r 2}=1, \sigma_{2}=20$ $\mathrm{S} / \mathrm{m}$. The field computation by the in-house full wave solver is validated against FEKO and the relative error (blue curve) are found to be less than 1\% (grey dashed line).

\section{A. Horizontal Embedded CNT}

The schematic diagram of a horizontal embedded CNT in lossy three-layer media was shown previously in Fig. 2a. For validation, we consider a simple CNT composite, where the top and bottom layer is considered as air, the middle layer is $d$ $=1 \mu \mathrm{m}$ thick dielectric with relative permittivity $\varepsilon_{r 2}=10$ and conductivity $\sigma_{2}=1 \mathrm{~S} / \mathrm{m}$. The horizontal CNT is $l=100 \mathrm{~nm}$ long, with a radius $r_{c n t}=0.61 \mathrm{~nm}$, and placed at a height $z_{0}$ $=0.75 \mu \mathrm{m}$ from the bottom interface. The composite structure in Fig. 2a is illuminated by a transverse magnetic (TM) plane wave excitation normally incident on the top interface, i.e. with zero incidence angle $\left(\theta_{i}=0^{\circ}\right)$, so that the incident electric field has only $E_{x}$-component and couples maximally to the $\mathrm{x}$-directed CNT. Following the ATW condition (31), the in-house solver discretizes the embedded $100 \mathrm{~nm}$ long CNT into $S=25$ small equal segments and assigns frequency dependent complex distributed line impedance $\left(Z_{c n t}\right)$ to each

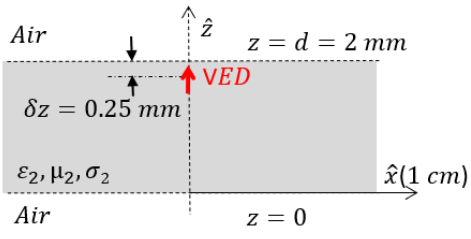

(a)
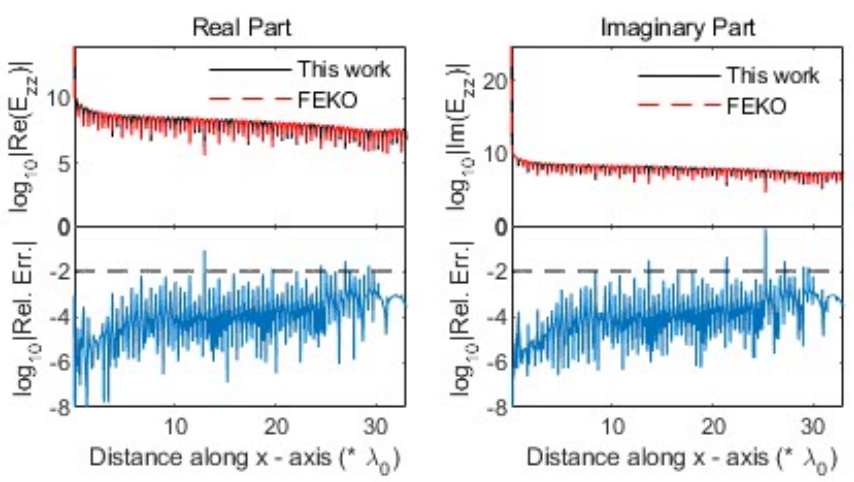

(b)
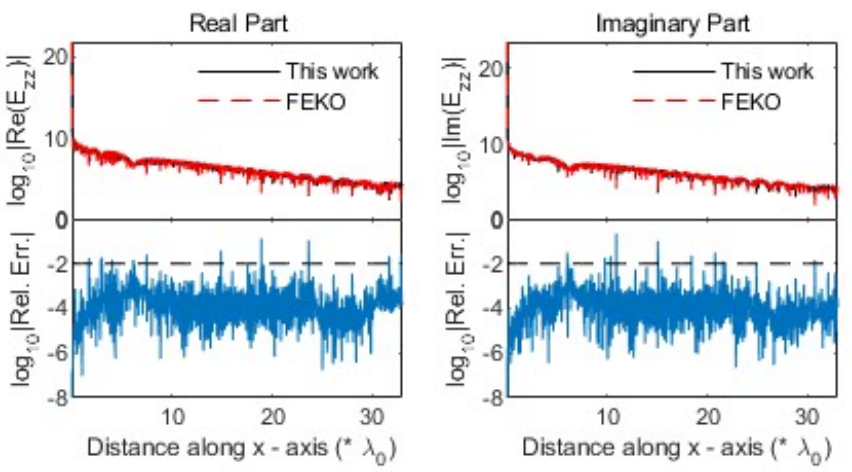

(c)

Fig. 6: (a) Schematic diagram of an $z$-directed electric dipole placed inside a $d=2 \mathrm{~mm}$ thick semi-infinite lossy dielectric slab at a depth of $\delta z=0.25 \mathrm{~mm}$ from the top interface. The top and bottom layers are considered as air. The $z$-component of the electric field is computed at the source height at $f=1 \mathrm{THz}\left(\lambda_{0}=3 \times 10^{-4} \mathrm{~m}\right)$ for a lateral range of $x=0.61 \mathrm{~nm}$ to $1 \mathrm{~cm}$, for the following two different lossy dielectric cases, (b) $\varepsilon_{r 2}=1.1, \mu_{r 2}=1, \sigma_{2}=10^{-9} \mathrm{~S} / \mathrm{m}$, (c) $\varepsilon_{r 2}=20, \mu_{r 2}=1, \sigma_{2}=20$ $\mathrm{S} / \mathrm{m}$. The field computation by the in-house full wave solver is validated against FEKO and the relative error (blue curve) are found to be less than 1\% (grey dashed line).

segment. No discretization is required for the layer as the inhouse solver uses multilayer DGFs to account for the layer effect. The absorbed power spectrum $\left(P_{a b s}\right)$ of the horizontal embedded CNT is computed by the in-house solver from 1 $\mathrm{THz}$ to $10 \mathrm{THz}$ and is shown as the black curve in Fig. 7. The $100 \mathrm{~nm}$ long horizontal embedded CNT shows resonance at $6.9 \mathrm{THz}$. The unique complex conductivity of CNT and the presence of the layered media allow the CNT to resonate at a much lower frequency than a similar sized PEC wire in free space. For example, the first resonance of an isolated $100 \mathrm{~nm}$ long PEC wire in free space appears near $1500 \mathrm{THz}$, and that of a $100 \mathrm{~nm}$ long CNT in free space appears near 22 $\mathrm{THz}$, irrespective of their orientation [13]. While embedded in a dielectric layer the same CNT resonates at a much lower frequency value depending on the layer characteristics.

We validate the in-house solver results against the MoM based FEKO planar multilayer substrate solver, which uses a 


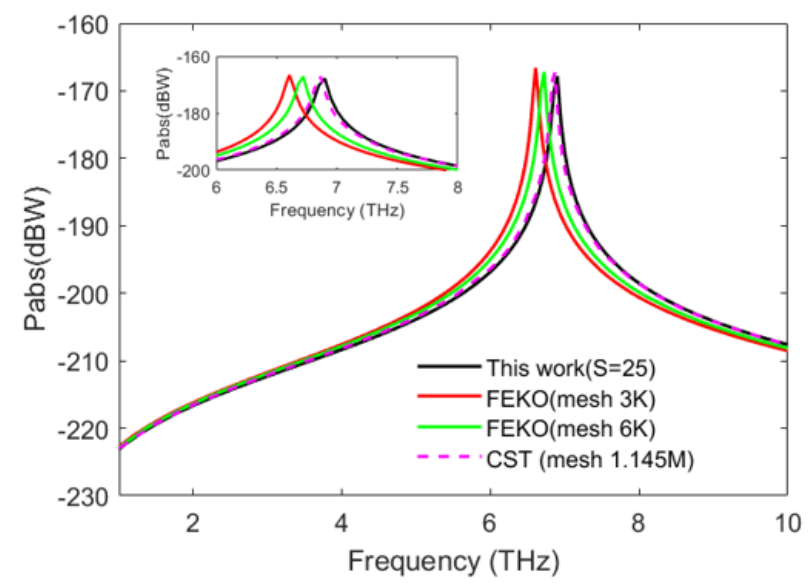

Fig. 7: Validation of absorbed power spectrum of an embedded horizontal CNT (see Fig. 2a) using three different solvers: (1) MoM based 1D in-house solver (this work), (2) MoM based 3D FEKO solver, (3) FEM based CST MWS. Composite parameters are as follows: $\varepsilon_{r 1}=\varepsilon_{r 3}=1, \varepsilon_{r 2}=10, \mu_{r 1}=\mu_{r 2}=\mu_{r 3}=1, \sigma_{1}=\sigma_{3}=$ $0, \sigma_{2}=1 \mathrm{~S} / \mathrm{m}, d=1 \mu \mathrm{m}, z_{0}=0.75 \mu \mathrm{m}, l=100 \mathrm{~nm}, r_{c n t}=0.61 \mathrm{~nm}$. TM wave excitation with $\theta=0^{\circ}$.

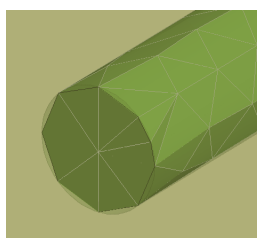

(a)

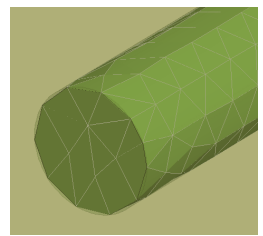

(b)

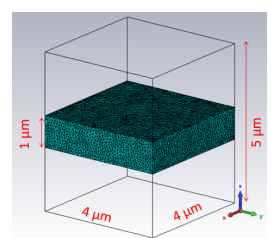

(c)
Fig. 8: Three-dimensional (3D) full-wave model in commercial solvers for a $100 \mathrm{~nm}$ long single CNT embedded in a $1 \mu \mathrm{m}$ thick dielectric slab. (a) Zoomed view of the 3D cylindrical model for CNT in FEKO MoM solver discretized by 3000 triangular surface mesh elements, and (b) discretized by 6000 triangular surface mesh elements The dielectric slab does not need discretization. (c) 3D unit cell in CST solver with periodic boundary condition assigned in $\mathrm{x}$ and $\mathrm{y}$ direction for the single CNT embedded in the dielectric slab where the whole setup is discretized by $1,145,026$ tetrahedral mesh elements.

similar multilayer Green's function approach that avoids layer discretization complexity [39]. However, FEKO does not allow assigning complex impedance to $1 \mathrm{D}$ wire structures, and thus the CNTs are modeled as 3D penetrable objects (cylinders), discretized by triangular surface mesh elements, and assigned a frequency dependent equivalent complex permittivity profile as given in Appendix A, Eq. (A.3). Since the CNTs are inherently high aspect ratio wire-like structures, a large number of surface mesh elements is required to accurately define the wire curvature of 3D cylindrical model. In the present example, the $100 \mathrm{~nm}$ long CNT with a radius $r_{c n t}=0.61 \mathrm{~nm}$ has an aspect ratio (length/diameter) $\approx 80$. To study the meshing effect on solution accuracy and compare the computation time we simulated different mesh densities for the 3D CNT structure in FEKO. Two such discretization in FEKO are shown by the zoomed view in Fig. 8a (using 3000 mesh elements) and Fig. $8 b$ (using 6000 mesh elements). The FEKO results show overall good agreement with the in-house results except for the small red-shifts in resonance frequency (Fig. 7 red and green curve). We find that the red-shift decreases from $4.35 \%$ to $2.61 \%$, when the CNT surface mesh elements assigned by FEKO are doubled from 3000 to 6000 triangles. That means with increasing meshing density, the 3D CNT resonance should converge to the 1D CNT resonance. This is because increasing mesh elements in FEKO better approximates the wire curvature and thus we get increasingly accurate 3D MoM solutions. It is obvious that the 3D meshed cylindrical shape will always remain crude compared to the actual cylindrical shape (which ideally would need an infinite number of mesh elements), and thus a small but finite red-shift will always persist in the FEKO generated resonance as compared to the in-house solution. This observation is in accordance to our previously reported work [13], where the CNTs were investigated in a free space condition. To better visualize the convergence phenomena of embedded CNT resonance we plot both the FEKO resonance and the in-house resonance with increasing mesh density in Fig. 9. The top x-axis shows inverse of the number of linear segments used by the in-house solver to discretize the 1D CNT and corresponding resonances are plotted in black. The bottom x-axis shows inverse of the square root of number of triangles used by FEKO to discretize the 3D CNT surface and corresponding resonances are plotted in red. For both the top and bottom $\mathrm{x}$-axis, mesh density increases towards the left. The black curve shows that the in-house resonance remains unchanged when we use more than 20 segments for $100 \mathrm{~nm}$ long CNT. However the red curve shows that the FEKO resonance frequency value gradually rises as the triangular mesh density increases from 3000 , to 4500 , to 6000 . The extrapolated red curve shows that the FEKO will need more than 20,000 triangular mesh elements to approximately merge with the in-house resonance.

To further confirm the results of Fig. 7 we performed an extra validation step. We chose finite element method (FEM) based CST Microwave studio [40], a full-wave electromagnetic solver that uses a totally different approach than the MoM based FEKO and the in-house solver. The FEM based CST solver requires discretization of the embedding layer surfaces as well as the embedded 3D CNT with large number of tetrahedral mesh elements. As shown in Fig. 8c, we design the $1 \mu \mathrm{m}$ thick dielectric slab with embedded CNT confined in a 3D unit cell of $4 \mu \mathrm{m} \times 4 \mu \mathrm{m} \times 5 \mu \mathrm{m}$. The $z_{\min / \max }$ are set to open boundary conditions to simulate air above and below the dielectric interfaces. Periodic boundary condition is applied on $x_{\min / \max }$ and $y_{\min / \max }$ to simulate infinite lateral extent of all three layers. We chose the adaptive mesh setting in CST and solved from $1 \mathrm{THz}$ to $10 \mathrm{THz}$. The CST solution slowly converges to the in-house solution of $P_{a b s}$ with increasing number of iterative passes. The CST computed $P_{a b s}$ is also plotted in Fig. 7 (magenta dashed curve), which shows excellent agreement with the in-house result. The CST resonance frequency value is less than $1 \%$ red-shifted compared to the in-house resonance frequency value. To achieve this result CST assigned a total of 1,145,026 tetrahedral mesh elements to discretize the confined composite structure in a unit cell. Thus it is evident that with increasing mesh density both the FEKO 3D MoM solution and CST 3D FEM solution converge towards our in-house 1D MoM solution of embedded CNT.

The disadvantage of increasing mesh density is the price of increased computation time. For a fair comparison, we only compared the time required by FEKO and our in-house 


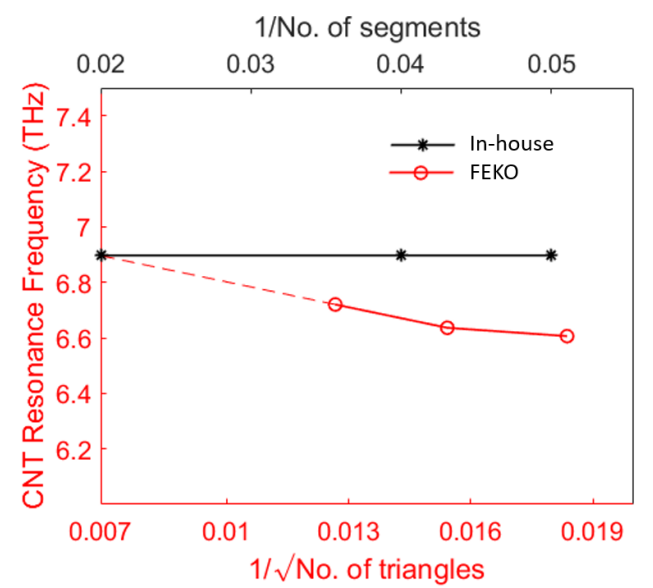

Fig. 9: Convergence of FEKO resonance towards the in-house solver resonance with increasing mesh density for an embedded $100 \mathrm{~nm}$ long horizontal CNT. The top x-axis shows the inverse of segment number used by in-house code for 1D CNT. The bottom $\mathrm{x}$-axis shows the inverse of square root of triangular mesh elements used by FEKO for 3D cylindrical CNT. Composite parameters remains same as in Fig. 7.

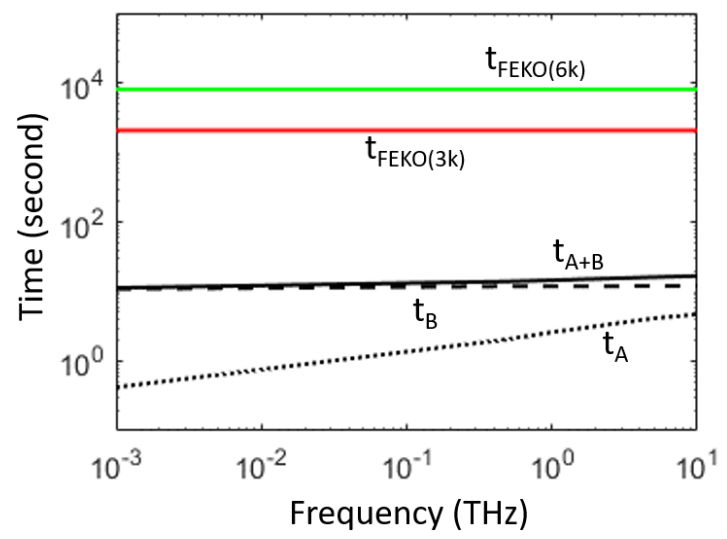

Fig. 10: Comparison of the $[Z]$ filling time required by in-house solver vs FEKO for the embedded horizontal $100 \mathrm{~nm}$ long CNT as described in Fig. 7.

solver. Both the solvers use multilayer Green's function based MoM technique that obviates the computational cost of layer discretization. We only consider the time to calculate and fill the impedance matrix at each operating frequency. An Intel(R) Xeon(R) CPU E5-2697A v4 @ $2.60 \mathrm{GHz}$ machine, with 512 GB installed RAM, was used to study the computation time. Since the in-house algorithm does not involve any parallel computation, FEKO was also operated in serial mode. Fig. 10 plots the CPU time (in second) elapsed until the $[Z]$ matrix is filled along the $y$-axis, and increasing frequency $(1 \mathrm{GHz}$ to $10 \mathrm{THz})$ along the $\mathrm{x}$-axis. The red solid line refers to the computation time required by the FEKO 3000 mesh $\left(t_{F E K O(3 K)}\right)$, and the green solid lines refers to the computation time required by the FEKO 6000 mesh $\left(t_{F E K O(6 K)}\right)$. The time required by the in-house algorithm (for $S=25$ segment CNT) is plotted as the black solid curve $\left(t_{A+B}\right)$, which is the sum of two independent times $t_{A}$ and $t_{B}$. $t_{A}$ is the time required to build the LUT for contour integration on the axial mesh grid along the CNT length. $t_{A}$ rises with increasing frequency as the upper limit of contour integration $k_{M}$ in (27) and (28) increases and more sampling points and iterations are needed to maintain a desired numerical accuracy.
However, $t_{B}$ is independent of frequency. $t_{B}$ is the combined time required to compute the closed form SI tail on the CNT axial mesh grid, followed by the interpolation of the full-wave solution and filling of the $[Z]$ matrix.

Observing the entire frequency range in Fig. 10, we see that $t_{F E K O(3 K)} \approx 2000 \mathrm{~s}$, and $t_{F E K O(6 K)} \approx 8000 \mathrm{~s}$. Thus to reduce the resonant frequency red-shift approximately by a factor of two (from $2.61 \%$ to $4.35 \%$ ) FEKO needs a two fold increase in surface mesh density (from 3000 to 6000), which in turn increase the computation time by four fold (from 2000 $\mathrm{s}$ to $8000 \mathrm{~s}$ ). However, to solve a $[Z]_{24 \times 24}$ matrix for the horizontal CNT, the in-house solver takes only $t_{A+B}=11.5$ $\mathrm{s} @ 1 \mathrm{GHz}$, and $t_{A+B}=16.7 \mathrm{~s} @ 10 \mathrm{THz}$. Thus we achieve on average a computation speed up of $\frac{t_{F E K O(6 \mathrm{~K})}}{t_{A+B}} \approx 570$ for a $100 \mathrm{~nm}$ long horizontal embedded CNT while using the inhouse solver as compared to FEKO that still suffers from a $2.61 \%$ red-shift in resonant frequency.

\section{B. Vertical Embedded CNT}

To perform similar full-wave analysis with a vertical embedded CNT we keep every other composite parameter the same as mentioned in Fig. 7, except for the CNT orientation and incident excitation. The schematic diagram of the vertical embedded CNT in lossy three-layer media was shown previously in Fig. $2 \mathrm{~b}$ where the CNT was oriented along $\mathrm{z}$-axis and centered at a height $z_{0}=0.75 \mu \mathrm{m}$ from the bottom interface. The vertical embedded CNT (Fig. 2b) is illuminated by a transverse electric (TE) plane wave excitation with oblique incidence $\left(\theta_{i}=80^{\circ}\right)$, which has a large $E_{z}$ component that couples to the z-directed CNT.

The absorbed power spectrum $\left(P_{a b s}\right)$ for the embedded vertical CNT is computed using the in-house solver and FEKO from $1 \mathrm{THz}$ to $10 \mathrm{THz}$ and the results are compared in Fig. 11. For ease of comparison, we keep the mesh settings similar to the previously discussed horizontal CNT case. From the in-house calculation, we find that the $100 \mathrm{~nm}$ long vertical embedded CNT also resonates at $6.9 \mathrm{THz}$, similar to the embedded horizontal CNT. However due to the difference in incident electric field polarization and CNT orientation the absorbed power spectrum of the vertical embedded CNT (Fig. 11) is found to be approximately $19 \mathrm{~dB}$ lower than that of the horizontal embedded CNT (Fig. 7). The FEKO simulation results for the vertical embedded CNT with 3000 and 6000 mesh shows good agreement with the in-house results (Fig. 11). In this case also we find small red-shifts in resonance frequency exhibited by FEKO as compared to the in-house solver. The red-shift decreases from $4.35 \%$ to $2.61 \%$, when the number of FEKO surface mesh elements are doubled from 3000 to 6000 triangles.

Using the same Intel(R) Xeon(R) CPU E5-2697A v4 @ 2.60 GHz machine, with $512 \mathrm{~GB}$ installed RAM, we record the computation time required to solve the vertical embedded CNT. Fig. 12 shows the time comparison between FEKO and the in-house solver. The $y$-axis indicates the CPU time (in seconds) elapsed until the $[Z]$ matrix is filled, and the $\mathrm{x}$-axis shows increasing frequency from $1 \mathrm{GHz}$ to $10 \mathrm{THz}$. The definition of $t_{F E K O(3 K)}, t_{F E K O(6 K)}, t_{A}, t_{B}$ and $t_{A+B}$ 


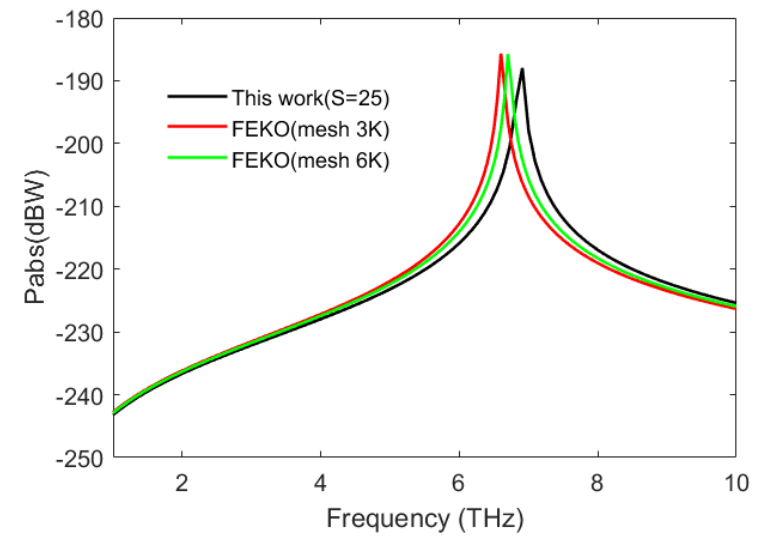

Fig. 11: Validation of absorbed power spectrum of an embedded vertical CNT (see Fig. 2b) using MoM based 1D in-house solver (this work) and MoM based 3D FEKO solver. Composite parameters are as follows: $\varepsilon_{r 1}=\varepsilon_{r 3}=1, \varepsilon_{r 2}=10, \mu_{r 1}=\mu_{r 2}=$ $\mu_{r 3}=1, \sigma_{1}=\sigma_{3}=0, \sigma_{2}=1 \mathrm{~S} / \mathrm{m}, d=1 \mu \mathrm{m}, z_{0}=0.75 \mu \mathrm{m}, l=100 \mathrm{~nm}, r_{c n t}$ $=0.61 \mathrm{~nm}$. TE wave excitation with $\theta=80^{\circ}$.

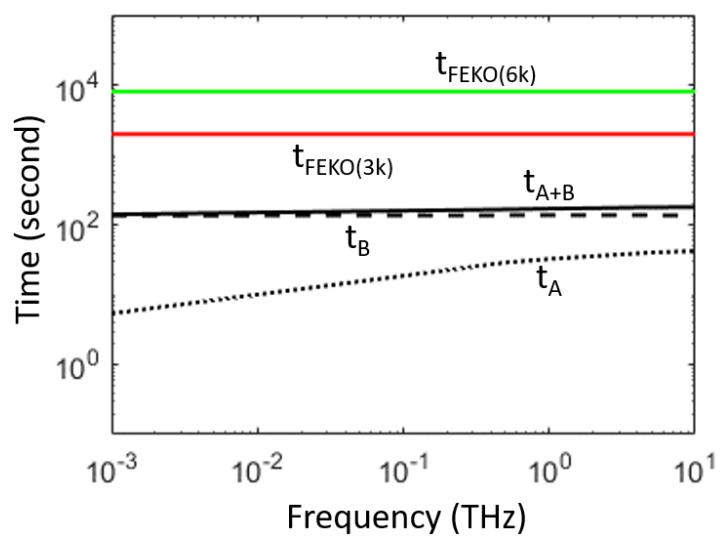

Fig. 12: Comparison of the $[Z]$ filling time required by in-house solver vs FEKO for the embedded vertical $100 \mathrm{~nm}$ long CNT as described in Fig. 11

remains same as in Fig. 10. To solve a $[Z]_{24 \times 24}$ matrix for the vertical embedded CNT, the in-house solver takes $t_{A+B}$ $=140.2 \mathrm{~s} @ 1 \mathrm{GHz}$, and $t_{A+B}=177.3 \mathrm{~s} @ 10 \mathrm{THz}$ as shown in Fig. 12. This is almost ten times more than what was observed in Fig. 10 for the horizontal CNT. To explain this ten fold increase in computation time we can refer back to the last paragraph of section II-D, where it was discussed that for the horizontal CNT an 1D LUT is employed considering its lateral invariance property, but for the vertical CNT a 2D LUT is required. The FEKO solution time for the embedded 3D CNT remains similar irrespective of any CNT orientation. This is because FEKO fetches Green's function data from a pre-formatted built-in LUT that works in similar fashion for any orientation of the 3D object. However, for the $100 \mathrm{~nm}$ long vertical embedded CNT we still achieve an average computation speed up of $\frac{t_{F E K O(6 K)}}{t_{A+B}} \approx 50$ by using the inhouse solver as compared to FEKO.

\section{CONCLuSion}

A novel full wave method of moment (MoM) solver was developed for accurate and efficient non-invasive evaluation of the electromagnetic response from multiscale CNT reinforced composites. The three dimensional (3D) multiscale composite problem has been effectively reduced to a one dimensional (1D) arbitrary thin wire problem by using the multilayer dyadic Green's function (DGF) which accurately accounts for the lossy substrate effect. A modified semi-analytical technique was conceived for fast evaluation of the associated Sommerfeld type integrals. The integrand singularities were avoided by adaptive contour deformation. The slow converging integrand tails were computed in exact closed form. The reliability of the in-house solver was tested for a wide range of composite parameters, including $1 \mathrm{GHz}$ to 10 $\mathrm{THz}$ operating frequency, $\mathrm{nm}$ to $\mathrm{mm}$ thick substrate, $\mathrm{nm}$ to $\mathrm{cm}$ range of lateral separation between source and observation point, and also a wide variety of media properties to cover most CNT composite applications. The accuracy of the in-house algorithm was verified rigorously against multiple commercial 3D full-wave solver. The absorbed power spectrum of horizontal and vertical embedded CNTs are studied. It is observed that, with increasing mesh density, the results achieved by the $3 \mathrm{D}$ commercial solvers gradually converge towards the results achieved by the solver proposed in this work. The in-house algorithm (in serial mode) solves a $100 \mathrm{~nm}$ long embedded CNT $\approx 570$ times faster for horizontal orientation, and $\approx 50$ times faster for vertical orientation as compared to the FEKO 3D MoM solver. Thus for composite problems the present approach will be more scalable than available commercial full-wave solvers. In its present form, the solver can be rigorously applied to investigate horizontal and vertical arrangements of embedded CNTs and study the effects of composite parameters on the total electromagnetic response. The algorithm is currently being expanded to include additional DGF components. Parallelization of the algorithm can further improve the solver speed.

In summary, the proposed full-wave solver overcomes the limitations of: (i) the dilute limit effective media approximations (EMA) which are fast but inaccurate beyond low frequencies, and (ii) 3D full wave commercial solvers (FEM, MoM) which give comparable accuracy but at the expense of enormous computation resources and time. The proposed solver could enable the simulations of more complex composite structures that are engineered to produce tailored electromagnetic behavior. Also, the greatly improved computational efficiency of the in-house algorithm will be very useful for real-time interpretation of the spectroscopy data to facilitate the non-destructive evaluation (NDE) of CNT composites.

\section{APPENDIX A}

\section{Electrical Properties of Single WALLEd CNTS}

The ATW CNTs are assigned Drude-like axial surface conductivity as follows [30],

$$
\sigma_{c n t}=\frac{\sigma_{0}}{1+j \omega \tau}, \sigma_{0}=\frac{2 e^{2} \nu_{F} \tau}{2 \pi \hbar r_{c n t}}
$$

where $\sigma_{0}$ is the CNT static DC conductivity, $\tau=0.3 \mathrm{ps}$ to $3 \mathrm{ps}$ is the relaxation time, $e$ is the electronic charge, $\nu_{F}=$ 
$9.71 \times 10^{5} \mathrm{~m} / \mathrm{s}$ is the Fermi velocity, $\hbar$ is Plank's constant divided by $2 \pi$, and $r_{c n t}$ is the CNT radius. The axial surface conductivity in (A.1) is valid in the microwave through 100 THz range for metallic CNTs with small chirality $(n, m)<50$ [41]. In this study, we have considered only single walled CNTs (SWCNTs) with a $(9,9)$ armchair chirality resulting in a cross-sectional CNT radius of $r_{c n t}=0.61 \mathrm{~nm}$. The relaxation time was set to $\tau=3 \mathrm{ps}$ to generate sharp resonances in power spectrum. The CNT axial surface conductivity can be translated into a one-dimensional distributed complex impedance $Z_{c n t}$ as follows [13]:

$$
Z_{c n t}=R_{c n t}+j \omega L_{c n t}=\frac{1}{2 \pi r_{c n t} \sigma_{c n t}}
$$

where,

$$
R_{c n t}=\frac{1}{2 \pi r_{c n t} \sigma_{0}}, L_{c n t}=\frac{\tau}{2 \pi r_{c n t} \sigma_{0}}
$$

$R_{c n t}$ is the Ohmic distributed resistance and $L_{c n t}$ is the kinetic inductance of the CNT.

While modeling the CNT as a penetrable 3D cylindrical structure in FEKO, the region inside the cylinder was assigned an appropriate complex permittivity profile following the below steps,

$$
\left\{\begin{array}{c}
\sigma_{c y l}=\frac{1}{Z_{c n t} \pi r_{c n t}^{2}} \\
\varepsilon_{r_{c y l}}=\frac{\mathcal{I} m\left(\sigma_{c y l}\right)-j \mathcal{R} e\left(\sigma_{c y l}\right)}{\omega \varepsilon_{0}} \\
\tan \delta_{c y l}=-\frac{\mathcal{I} m\left(\varepsilon_{r_{c y l}}\right)}{\mathcal{R} e\left(\varepsilon_{r_{c y l}}\right)}
\end{array}\right.
$$

\section{APPENDIX B \\ SOMMERFELD IDENTITIES}

The following exact closed-form solutions of Sommerfeld type integrals are derived using the Fourier-Bessel transform relationships given in [57].

$$
S_{0}=\int_{0}^{\infty} d \lambda\left[\lambda J_{0}(\lambda r)\right] \frac{e^{-a_{n} \sqrt{\lambda^{2}-k_{i}^{2}}}}{\sqrt{\lambda^{2}-k_{i}^{2}}}=\frac{e^{-j k_{i} A}}{A}
$$

where $A=\sqrt{r^{2}+a_{n}^{2}}$.

$$
\begin{aligned}
S_{1} & =\int_{0}^{\infty} d \lambda\left[\frac{J_{1}(\lambda r)}{r}\right] \frac{e^{-a_{n} \sqrt{\lambda^{2}-k_{i}^{2}}}}{\sqrt{\lambda^{2}-k_{i}^{2}}}=\frac{e^{-j k_{i} a_{n}}-e^{-j k_{i} A}}{A} \\
S_{2} & =\frac{\partial^{2}}{\partial a_{n}^{2}}\left\{S_{0}\right\}=\int_{0}^{\infty} d \lambda\left[\lambda J_{0}(\lambda r)\right] \sqrt{\lambda^{2}-k_{i}^{2}} e^{-a_{n} \sqrt{\lambda^{2}-k_{i}^{2}}} \\
& =\frac{e^{-j k_{i} A}}{A^{2}}\left[-j k_{i}-\frac{1+k_{i}^{2} a_{n}^{2}}{A}+\frac{3 j k_{i} a_{n}^{2}}{A^{2}}+\frac{3 a_{n}^{2}}{A^{3}}\right] \\
S_{3} & =\frac{\partial^{2}}{\partial a_{n}^{2}}\left\{S_{1}\right\}=\int_{0}^{\infty} d \lambda\left[\frac{J_{1}(\lambda r)}{r}\right] \sqrt{\lambda^{2}-k_{i}^{2}} e^{-a_{n} \sqrt{\lambda^{2}-k_{i}^{2}}} \\
& =\frac{1}{r^{2}}\left[j k_{i} e^{-j k_{i} a_{n}}+\frac{e^{-j k_{i} A}}{A}\left(1-\frac{j k_{i} a_{n}^{2}}{A}-\frac{a_{n}^{2}}{A^{2}}\right)\right]
\end{aligned}
$$

$$
\begin{aligned}
S_{4} & =-\frac{\partial^{2}}{\partial r^{2}}\left\{S_{0}\right\}-\frac{1}{r} \frac{\partial}{\partial r}\left\{S_{0}\right\} \\
& =\int_{0}^{\infty} d \lambda\left[\lambda^{3} J_{0}(\lambda r)\right] \frac{e^{-a_{n} \sqrt{\lambda^{2}-k_{i}^{2}}}}{\sqrt{\lambda^{2}-k_{i}^{2}}} \\
& =\frac{e^{-j k_{i} A}}{A^{2}}\left[2 j k_{i}+\frac{2-\left(j k_{i} r\right)^{2}}{A}-\frac{3 j k_{i} r^{2}}{A^{2}}-\frac{3 r^{2}}{A^{3}}\right]
\end{aligned}
$$

\section{REFERENCES}

[1] M. F. L. De Volder, S. H. Tawfick, R. H. Baughman, and A. J. Hart, "Carbon nanotubes: Present and future commercial applications," Science, vol. 339, no. 6119, pp. 535-539, 2013.

[2] A. Kausar, I. Rafique, and B. Muhammad, "Review of applications of polymer/carbon nanotubes and epoxy/cnt composites," Polymer-Plastics Technology and Engineering, vol. 55, no. 11, pp. 1167-1191, 2016.

[3] S. Kholghi Eshkalak, A. Chinnappan, W. Jayathilaka, M. Khatibzadeh, E. Kowsari, and S. Ramakrishna, "A review on inkjet printing of cnt composites for smart applications," Applied Materials Today, vol. 9, pp. 372-386, 2017

[4] S. Lu, J. Shao, K. Ma, D. Chen, X. Wang, L. Zhang, Q. Meng, and J. Ma, "Flexible, mechanically resilient carbon nanotube composite films for high-efficiency electromagnetic interference shielding," Carbon, vol. 136, pp. 387-394, 2018.

[5] C. Liu, I. Sergeichev, I. Akhatov, and K. Lafdi, "Cnt and polyaniline based sensors for the detection of acid penetration in polymer composite," Composites Science and Technology, vol. 159, pp. 111-118, 2018.

[6] L. Wang and Z.-M. Dang, "Carbon nanotube composites with high dielectric constant at low percolation threshold," Applied Physics Letters, vol. 87, no. 4, p. 042903, 2005.

[7] Z.-M. Dang, L. Wang, Y. Yin, Q. Zhang, and Q.-Q. Lei, "Giant dielectric permittivities in functionalized carbon-nanotube/ electroactive-polymer nanocomposites," Advanced Materials, vol. 19, no. 6, pp. 852-857, 2007.

[8] Y. Wang, S. Kim, G. Li, and L. Sun, "Filler orientation effect on relative permittivity of dielectric elastomer nanocomposites filled with carbon nanotubes," Computational Materials Science, vol. 104, pp. 69$75,2015$.

[9] A. M. Hassan and E. J. Garboczi, "Electromagnetic scattering from randomly-centered parallel single-walled carbon nanotubes embedded in a dielectric slab," IEEE Transactions on Antennas and Propagation, vol. 62, no. 10, pp. 5230-5241, Oct 2014.

[10] R. Simoes, J. Silva, R. Vaia, V. Sencadas, P. Costa, J. Gomes, and S. Lanceros-Méndez, "Low percolation transitions in carbon nanotube networks dispersed in a polymer matrix: dielectric properties, simulations and experiments," Nanotechnology, vol. 20, no. 3, p. 035703 , dec 2008.

[11] A. M. Hassan, J. Obrzut, and E. J. Garboczi, "A Q-band free-space characterization of carbon nanotube composites," IEEE Transactions on Microwave Theory and Techniques, vol. 64, no. 11, pp. 3807-3819, Nov 2016.

[12] T. Morimoto, S.-K. Joung, T. Saito, D. N. Futaba, K. Hata, and T. Okazaki, "Length-dependent plasmon resonance in single-walled carbon nanotubes," ACS Nano, vol. 8, no. 10, pp. 9897-9904, 2014.

[13] S. Dey, E. J. Garboczi, and A. M. Hassan, "Electromagnetic resonance analysis of asymmetric carbon nanotube dimers for sensing applications," Nanotechnology, vol. 31, no. 42, p. 425501, aug 2020.

[14] M. Shuba, D. Yuko, P. Kuzhir, S. Maksimenko, M. Kanygin, A. Okotrub, R. Tenne, and P. Lambin, "How effectively do carbon nanotube inclusions contribute to the electromagnetic performance of a composite material? estimation criteria from microwave and terahertz measurements," Carbon, vol. 129, pp. 688-694, 2018.

[15] F. Vargas-Lara, A. M. Hassan, E. J. Garboczi, and J. F. Douglas, "Intrinsic conductivity of carbon nanotubes and graphene sheets having a realistic geometry," The Journal of Chemical Physics, vol. 143, no. 20, p. 204902, 2015.

[16] A. M. Hassan, F. Vargas-Lara, J. F. Douglas, and E. J. Garboczi, "Electromagnetic resonances of individual single-walled carbon nanotubes with realistic shapes: A characteristic modes approach," IEEE Transactions on Antennas and Propagation, vol. 64, no. 7, pp. 27432757, 2016.

[17] A. M. Hassan, F. Vargas-Lara, J. F. Douglas, and E. J. Garboczi, "Electromagnetic scattering from multiple single-walled carbon nanotubes having tumbleweed configurations," IEEE Transactions on Antennas and Propagation, vol. 65, no. 6, pp. 3192-3202, June 2017. 
[18] H. Zein and Y. A. Al-Shataif, "A state of the art review of dispersion and inspection techniques for carbon nanotubes (cnts) into matrix composites," Materials Transactions, vol. 61, no. 1, pp. 14-26, 2020.

[19] B. Natarajan, N. D. Orloff, R. Ashkar, S. Doshi, K. Twedt, A. Krishnamurthy, C. Davis, A. M. Forster, E. Thostenson, J. Obrzut, R. Sharma, and J. A. Liddle, "Multiscale metrologies for process optimization of carbon nanotube polymer composites," Carbon, vol. 108, pp. 381-393, 2016.

[20] C. J. Long, N. D. Orloff, K. A. Twedt, T. Lam, F. Vargas-Lara, M. Zhao, B. Natarajan, K. C. Scott, E. Marksz, T. Nguyen, J. F. Douglas, J. McClelland, E. Garboczi, J. Obrzut, and J. A. Liddle, "Giant surface conductivity enhancement in a carbon nanotube composite by ultraviolet light exposure," ACS Applied Materials \& Interfaces, vol. 8, no. 35, pp. $23230-23235,2016$

[21] P. C. Waterman and R. Truell, "Multiple scattering of waves," Journal of Mathematical Physics, vol. 2, no. 4, pp. 512-537, 1961.

[22] G. Y. Slepyan, M. V. Shuba, S. A. Maksimenko, C. Thomsen, and A. Lakhtakia, "Terahertz conductivity peak in composite materials containing carbon nanotubes: Theory and interpretation of experiment," Phys. Rev. B, vol. 81, p. 205423, May 2010.

[23] M. V. Shuba, A. V. Melnikov, A. G. Paddubskaya, P. P. Kuzhir S. A. Maksimenko, and C. Thomsen, "Role of finite-size effects in the microwave and subterahertz electromagnetic response of a multiwall carbon-nanotube-based composite: Theory and interpretation of experiments," Phys. Rev. B, vol. 88, p. 045436, Jul 2013.

[24] M. V. Shuba, A. G. Paddubskaya, P. P. Kuzhir, S. A. Maksimenko, G. Valusis, M. Ivanov, J. Banys, V. Ksenevich, and G. W. Hanson, "Observation of the microwave near-field enhancement effect in suspensions comprising single-walled carbon nanotubes," Materials Research Express, vol. 4, no. 7, p. 075033, jul 2017.

[25] Z. Dang, J. Yuan, J. Zha, T. Zhou, S. Li, and G. hua $\mathrm{Hu}$, "Fundamentals, processes and applications of high-permittivity polymer-matrix composites," Progress in Materials Science, vol. 57, pp. $660-723,2012$

[26] E. Dadrasnia, S. Puthukodan, and H. Lamela, "Terahertz electrical conductivity and optical characterization of composite nonaligned single- and multiwalled carbon nanotubes," Journal of Nanophotonics, vol. 8 , no. 1 , pp. $1-11,2014$.

[27] A. M. Hassan, M. K. Islam, S. On, B. Natarajan, I. Y. Stein, N. Lachman, E. Cohen, B. L. Wardle, R. Sharma, J. A. Liddle, and E. J. Garboczi, "Modeling the electromagnetic scattering characteristics of carbon nanotube composites characterized by 3-d tomographic transmission electron microscopy," IEEE Open Journal of Antennas and Propagation, vol. 1, pp. 142-158, 2020.

[28] D. H. S. Cheng, "On the formulation of the dyadic green's function in a layered medium," Electromagnetics, vol. 6, no. 2, pp. 171-182, 1986.

[29] C. Tai, Dyadic Green Functions in Electromagnetic Theory, United Kingdom : IEEE Press,1994.

[30] G. W. Hanson, "Fundamental transmitting properties of carbon nanotube antennas," IEEE Transactions on Antennas and Propagation, vol. 53, no. 11, pp. 3426-3435, Nov 2005.

[31] W. Gibson, The Method of Moments in Electromagnetics. London, U.K.: Chapman \& Hall, 2008.

[32] A. Sommerfeld, Partial Differential Equations in Physics, ser. ISSN. Elsevier Science, 1949.

[33] H. Karami, K. Sheshyekani, and F. Rachidi, "Mixed-potential integral equation for full-wave modeling of grounding systems buried in a lossy multilayer stratified ground," IEEE Transactions on Electromagnetic Compatibility, vol. 59, no. 5, pp. 1505-1513, Oct 2017.

[34] M. Paulus, P. Gay-Balmaz, and O. J. F. Martin, "Accurate and efficient computation of the green's tensor for stratified media," Phys. Rev. E, vol. 62, pp. 5797-5807, Oct 2000.

[35] P. Gay-Balmaz and J. R. Mosig, "Three-dimensional planar radiating structures in stratified media," International Journal of Microwave and Millimeter-Wave Computer-Aided Engineering, vol. 7, no. 5, pp. 330343, 1997.

[36] D. Chatterjee, S. Rao, and M. Kluskens, "Some new techniques for evaluating sommerfeld integrals for microstrip antenna analysis," in 2016 URSI international symposium on Electromagnetic theory (EMTS). IEEE, 2016, pp. 335-337.

[37] K. A. Michalski and J. R. Mosig, "Efficient computation of sommerfeld integral tails - methods and algorithms," Journal of Electromagnetic Waves and Applications, vol. 30, no. 3, pp. 281-317, 2016.

[38] MATLAB version (R2020a), The Mathworks, Inc., Natick, Massachusetts. [Online]. Available: https://www.mathworks.com/

[39] FEKO version (2019.3). [Online]. Available: https://www.altair.com/feko/
[40] CST Studio Suite (2020). [Online]. Available: https://www.3ds.com/products-services/simulia/products/cst-studiosuite/

[41] A. M. Nemilentsau, "Tutorial: Linear surface conductivity of an achiral single-wall carbon nanotube," Journal of Nanophotonics, vol. 5, no. 1, pp. $1-8,2011$.

[42] M. Abramowitz and I. Stegun, Handbook of Mathematical Functions: With Formulas, Graphs, and Mathematical Tables, ser. Applied mathematics series. Dover Publications, 1965.

[43] S. Barkeshli, P. H. Pathak, and M. Marin, "An asymptotic closed-form microstrip surface green's function for the efficient moment method analysis of mutual coupling in microstrip antennas," IEEE Transactions on Antennas and Propagation, vol. 38, no. 9, pp. 1374-1383, Sep. 1990

[44] Mengtao Yuan and T. K. Sarkar, "Computation of the sommerfeld integral tails using the matrix pencil method," IEEE Transactions on Antennas and Propagation, vol. 54, no. 4, pp. 1358-1362, April 2006.

[45] B. Wu and L. Tsang, "Fast computation of layered medium Green's functions of multilayers and lossy media using fast all-modes method and numerical modified steepest descent path method," IEEE Trans. Microw Theory Techn., vol. 56, no. 6, pp. 1446-1454, Jun. 2008.

[46] A. Alparslan, M. I. Aksun, and K. A. Michalski, "Closed-form green's functions in planar layered media for all ranges and materials," IEEE Transactions on Microwave Theory and Techniques, vol. 58, no. 3, pp. 602-613, March 2010.

[47] V. Arnautovski-Toseva and L. Grcev, "Image and exact models of a vertical wire penetrating a two-layered earth," IEEE Transactions on Electromagnetic Compatibility, vol. 53, no. 4, pp. 968-976, Nov 2011.

[48] E. Simsek, Qing Huo Liu, and Baojun Wei, "Singularity subtraction for evaluation of green's functions for multilayer media," IEEE Transactions on Microwave Theory and Techniques, vol. 54, no. 1, pp. 216-225, Jan 2006.

[49] D. Chatterjee, S. M. Rao, and M. S. Kluskens, "Improved evaluation of sommerfeld integrals for microstrip antenna problems," in 2013 International Symposium on Electromagnetic Theory, May 2013, pp. 981-984.

[50] P. Ding, C. Qiu, S. Zouhdi, and S. P. Yeo, "Rigorous derivation and fast solution of spatial-domain green's functions for uniaxial anisotropic multilayers using modified fast hankel transform method," IEEE Transactions on Microwave Theory and Techniques, vol. 60, no. 2, pp. 205-217, Feb 2012.

[51] J. Mosig, "The weighted averages algorithm revisited," IEEE Transactions on Antennas and Propagation, vol. 60, no. 4, pp. 20112018, April 2012.

[52] I. D. Koufogiannis, M. Mattes, and J. R. Mosig, "On the development and evaluation of spatial-domain green's functions for multilayered structures with conductive sheets," IEEE Transactions on Microwave Theory and Techniques, vol. 63, no. 1, pp. 20-29, Jan 2015.

[53] M. J. Neve and R. Paknys, "A technique for approximating the location of surface-and leaky-wave poles for a lossy dielectric slab," IEEE Trans. Antennas Propag., vol. 54, no. 1, pp. 115-120, Jan. 2006.

[54] K. C. Durbhakula, D. Chatterjee, and A. M. Hassan, "An efficient algorithm for locating te and tm poles for a class of multiscale inhomogeneous media problems," IEEE Journal on Multiscale and Multiphysics Computational Techniques, vol. 4, pp. 364-373, 2019.

[55] J. R. Mosig and K. A. Michaski, "Sommerfeld integrals and their relation to the development of planar microwave devices," IEEE Journal of Microwaves, vol. 1, no. 1, pp. 470-480, 2021.

[56] R. E. Collin, Field Theory of Guided Waves (second edition), NY, USA: IEEE Press, 1991, pp. 697-748.

[57] F. Oberhettinger, Tables of Bessel Transforms. Springer-Verlag, 1972. 